\title{
Large-Scale Microelectrode Recordings of High-Frequency Gamma Oscillations in Human Cortex during Sleep
}

\author{
Michel Le Van Quyen, ${ }^{1}$ Richard Staba, ${ }^{5}$ Anatol Bragin, ${ }^{5,9}$ Clayton Dickson, ${ }^{2,3,4}$ Mario Valderrama, ${ }^{1}$ Itzhak Fried, ${ }^{6}$ \\ and Jerome Engel ${ }^{5,7,8,9}$ \\ ${ }^{1}$ Centre de Recherche de l'Institut du Cerveau et de la Moelle Épinière-INSERM Unité Mixte de Recherche en Santé 975-Centre National de la Recherche \\ Scientifique Unité Mixte de Recherche 7225, Université Pierre et Marie Curie, Paris 6, Hôpital de la Pitié-Salpêtrière, 75651 Paris Cedex 13, France, \\ Departments of ${ }^{2}$ Psychology and ${ }^{3}$ Physiology, and ${ }^{4}$ Centre for Neuroscience, University of Alberta, Edmonton, T6G 2R3 Alberta, Canada, and Departments \\ of ${ }^{5}$ Neurology, ${ }^{6}$ Neurosurgery, ${ }^{7}$ Neurobiology, ${ }^{8}$ Psychiatry and Biobehavioral Science, and ${ }^{9}$ Brain Research Institute, David Geffen School of Medicine at \\ University of California, Los Angeles, Los Angeles, California 90095
}

Gamma oscillations $(40-120 \mathrm{~Hz})$, usually associated with waking functions, can be recorded in the deepest stages of sleep in animals. The full details of their large-scale coordination across multiple cortical networks are still unknown. Furthermore, it is not known whether oscillations with similar characteristics are also present in the human brain. In this study, we examined the existence of gamma oscillations during polysomnographically defined sleep-wake states using large-scale microelectrode recordings (up to 56 channels), with single-cell and spike-time precision, in epilepsy patients. We report that low $(40-80 \mathrm{~Hz})$ and high $(80-120 \mathrm{~Hz})$ gamma oscillations recurrently emerged over time windows of several hundreds of milliseconds in all investigated cortical areas during slow-wave sleep. These patterns were correlated with positive peaks of EEG slow oscillations and marked increases in local cellular discharges, suggesting that they were associated with cortical UP states. These gamma oscillations frequently appeared at approximately the same time in many different cortical areas, including homotopic regions, forming large spatial patterns. Coincident firings with millisecond precision were strongly enhanced during gamma oscillations but only between cells within the same cortical area. Furthermore, in a significant number of cases, cortical gamma oscillations tended to occur within $100 \mathrm{~ms}$ after hippocampal ripple/sharp wave complexes. These data confirm and extend earlier animal studies reporting that gamma oscillations are transiently expressed during UP states during sleep. We speculate that these high-frequency patterns briefly restore "microwake" activity and are important for consolidation of memory traces acquired during previous awake periods.

\section{Introduction}

Emerging evidence shows that sleep and wakefulness are not simply opposing brain states (Sejnowski and Destexhe, 2000; Steriade, 2000). In particular, gamma oscillations (40-120 Hz), usually associated with waking functions such as sensory binding (Singer and Gray, 1995), attention (Fries et al., 2001), encoding and retrieval of memory traces (Montgomery and Buzsáki, 2007), are also present during slow-wave sleep (SWS). In vivo (Steriade et al., 1996; Grenier et al., 2001; Isomura et al., 2006; Mukovski et al., 2007; Mena-Segovia et al., 2008) and in vitro (Dickson et al., 2003; Compte et al., 2008) recordings in the neocortex indicate that gamma oscillations occur during "UP" states, i.e., rhythmic cycles of suprathreshold membrane potential depolarizations occurring synchronously in large neuronal populations and reflected on electroencephalography (EEG) recordings as largeamplitude slow waves (Steriade et al., 1993). Network dynamics during UP states have been proposed to be equivalent to those

\footnotetext{
Received 0ct. 9, 2009; revised March 11, 2010; accepted April 6, 2010.

This work was supported by National Institutes of Health Grants NS 02808 and NS 33310.

Correspondence should be addressed to Michel Le Van Quyen, Centre de Recherche de l'Institut du Cerveau et de la Moelle Épinière INSERM Unité Mixte de Recherche en Santé 975-Centre National de la Recherche Scientifique Unité Mixte de Recherche 7225, Hôpital de la Pitié-Salpêtrière, 47 Boulevard de I'Hôpital, 75651 Paris Cedex 13, France. E-mail: lenalm@ext.jussieu.fr.

DOI:10.1523/JNEUROSCI.5049-09.2010

Copyright $\odot 2010$ the authors $\quad 0270-6474 / 10 / 307770-13 \$ 15.00 / 0$
}

observed during the waking state (Destexhe et al., 2007; Luczak et al., 2007; Haider and McCormick, 2009). In particular, during these highly dynamic states, gamma frequency fluctuations in inhibitory and excitatory synaptic potentials determine the timing of action potential generation at the millisecond level (Nowak et al., 1997; Hasenstaub et al., 2005), confirming that these oscillations may control the flow of communication across remote subpopulations of cells (Chrobak and Buzsáki, 1998; Salinas and Sejnowski, 2001; Fries, 2005; Haider and McCormick, 2009). Despite considerable understanding of cellular/synaptic mechanisms underlying gamma oscillations (Bartos et al., 2007), in vivo studies at the largescale level are still necessary to further characterize the full details of their local and long-range synchrony across vast cortical territories.

Studying large-scale functional dynamics of cortical networks in vivo is challenging and requires simultaneously recording local field potentials from multiple brain areas along with concurrently recorded neurons from each of these regions (Buzsáki, 2004). This system-level analysis of large-scale neuronal cooperation has only lately been able to be achieved, largely due to the technical difficulties. With recent advances in bioengineering, chronically implanted arrays of microelectrodes are an increasingly common tool for recording many neurons from various mammalian species (Fried et al., 1997; Nicolelis et al., 2003; Buzsáki, 2004; Cash et al., 2009). Nevertheless, the small size of a rat, for example, has greatly constrained the expanse of different 
cortical sites that can be studied using traditional microelectrode arrays. Furthermore, there is a paucity of data that exists between the levels of analysis that include single-unit ensemble recordings in animals and human scalp EEG.

In this study, believed to be the first quantitative, large-scale microelectrode recording in human cortex, we examined gamma patterns during sleep using multiple microwires (up to 56) in patients with epilepsy who required presurgical clinical evaluation. These microelectrodes provided a unique opportunity to study gamma oscillations directly adjacent to local cortical generators together with multiunit and single-unit activities, all in parallel with macroscopic scalp EEG. Furthermore, multiple sites can be arranged over larger distances, thus allowing for the simultaneous recording of local fields and units across multiple cortical regions.

\section{Materials and Methods}

Database. Subjects were nine patients with pharmacologically intractable epilepsy who were implanted with 8-14 intracranial depth electrodes to localize epileptogenic regions for possible resection (supplemental Table 1 , available at www.jneurosci.org as supplemental material). The placement of the electrodes was determined exclusively by clinical criteria (Fried et al., 1997). Extending beyond the tip of each electrode was nine Pt-Ir microwires ( $40 \mu \mathrm{m}$ diameter) that protruded $\sim 4 \mathrm{~mm}$ into the target tissue. Microwire tips randomly spread out into the target tissue in a cone shape with a minimal intertip spacing of $500 \mu \mathrm{m}$. The first eight microwires were insulated except for their tip and were used to record single-unit action potentials and local field potentials (LFPs). The ninth microwire had its insulation stripped for $\sim 1 \mathrm{~cm}$ and served as the recording reference for the other eight microwires on that depth probe. Signals from each microwire were amplified (gain $=10,000$ ), digitally sampled at $27.8 \mathrm{kHz}$, and bandpass filtered between $1 \mathrm{~Hz}$ and $6 \mathrm{kHz}$ (Cheetah recording system, Neuralynx). Spatial localizations were determined on the basis of postimplant computed tomography scans coregistered with preimplant 1.5 T MRI scans. Our results are based on microelectrode recordings located in the following cortical areas: posterior parahippocampal gyrus ( $n=136$ channels in 8 subjects), posterior and anterior cingulate cortex ( $n=68$ channels in 5 subjects), entorhinal cortex ( $n=$ 48 channels in 4 subjects), superior and anterior occipital cortex $(n=32$ channels in 2 subjects), superior temporal gyrus ( $n=16$ channels in 2 subjects), orbitofrontal cortex ( $n=16$ channels in 2 subjects), and supplementary motor area ( $n=8$ channels in 1 subject). To examine the impact of hippocampal discharges (epileptic spikes or sharp waves), recording sites within hippocampus were selected in five subjects $(1-3,6$, and 8 ), ipsilaterally to side of seizure onset ( $n=48$ channels). Spikes, by definition, have a duration shorter than $70 \mathrm{~ms}$, whereas sharp waves have a duration between 70 and $200 \mathrm{~ms}$ (de Curtis and Avanzini, 2001). In three of these subjects (1-3), simultaneous EEG recordings of the epileptic hippocampus and parahippocampal gyrus were performed using intracranial macroelectrodes with individual contact area of $6.5 \mathrm{~mm}^{2}$ and a sampling rate of $2000 \mathrm{~Hz}$ to characterize epileptiform spikes with standard clinical criteria. EEG, electrooculogram, and chin electromyogram activity were concurrently monitored and used to classify the different sleep states (Rechtschaffen and Kales, 1968). The recording states were quiet wakefulness, slow-wave sleep (stages 1-4), and rapid eye movement (REM) sleep. With the exception of three patients (subjects 1, 7, and 8 ), seizures did not occur within $12 \mathrm{~h}$ (or more) of the start of the sleep recordings (supplemental Table 1, available at www.jneurosci.org as supplemental material).

Automatic detection of high-frequency oscillations. Channels demonstrating oscillations with large-amplitude sinusoid-like waves with frequencies between 40 and $120 \mathrm{~Hz}$ that were discernable above background were selected for analysis. An automatic detection of gamma episodes was then achieved as described previously (Staba et al., 2004). In brief, we defined oscillatory events by detecting significant deviations of the envelope in the high-frequency range, longer than a minimal duration. The Hilbert transform was used to calculate the envelope of the filtered sig- nals between 40 and $120 \mathrm{~Hz}$. The duration threshold was set to $100 \mathrm{~ms}$ (i.e., 4-8 cycles of gamma oscillations) and the amplitude threshold was set to 3 SDs of the envelope calculated over the entire length of the signal. All the traces are then aligned at the beginning of the oscillation episode defined where the envelope crossed a threshold at 3 times the SD. The qualitative nature of our results was insensitive to the precise choice of thresholds from 3 to $5 \mathrm{SDs}$. With the current application, false-positive detections can be generated by harmonics of lower-frequency activities or by sharp components of action potentials that usually induce a broad band increase in the high-frequency range that obscured oscillation detection. For this purpose, we developed a custom graphical user interface (MATLAB) to visually screen candidate gamma oscillations. This interface permits us to display simultaneously raw signal, the bandpass-filtered signals, and a time-frequency map using a wavelet decomposition for frequency from 20 to $160 \mathrm{~Hz}$ (see next paragraph). Using this interface, we selected gamma events according to following criteria: (1) gamma oscillations had to be visually detectable on the unfiltered signals as sinusoidal waves; (2) the time-frequency map of a gamma oscillation had to show a primary peak in the frequency range $40-130 \mathrm{~Hz}$; and (3) gamma oscillations were not associated with epileptiform activity (chronic focal slowing or interictal spikes) in the same channel or a neighboring channel within a time window of $200 \mathrm{~ms}$. For the detection of ripples $(130-250 \mathrm{~Hz})$ and fast ripples $(250-600 \mathrm{~Hz})$, the same strategy was applied separately on 14 subsequent sub-bands of $40 \mathrm{~Hz}$ steps, ranging from 40 to $600 \mathrm{~Hz}$. In the cases of hippocampal recordings, interictal discharges (epileptic spikes or sharp waves) were not discarded to examine their temporal relationship with the detected high-frequency oscillations.

Oscillation analysis. A wavelet time-frequency analysis was used to determine precisely the mean frequency, the beginning, maximum amplitude, and onset and offset of each oscillation. The wavelet decomposition tool works as a mathematical microscope (Le Van Quyen and Bragin, 2007) that dissects the instantaneous frequency content of signal and enhances short-duration, low-amplitude activities, often masked by high-amplitude, low-frequency, and large-scale integrated field activity. The Morlet wavelet was here applied that uses a wave-like scalable function that is well localized in both time and frequency (see supplemental material, available at www.jneurosci.org). As a criterion of the significance for the time-frequency representations, we required the timefrequency peak energy to exceed the mean +3 SDs of baseline.

Spike sorting. All channels were high-pass filtered at $300 \mathrm{~Hz}$ and were visually examined for the presence of unit activities. In those microwires with clear unit activities, we performed spike detection ( $>4$ :1 signal-tonoise ratio) to obtain multiunit activities (MUAs). Single-unit activities were extracted with spike sorting using KlustaKwik 1.7 program (Harris et al., 2001) (software: http://klustakwik.sourceforge.net/), which employs the 10 principal components of the spike shape and an unsupervised CEM (conditional expectation maximization) clustering algorithm. After automatic clustering, the clusters containing nonspike waveforms were visually deleted, and then the units were further isolated using a manual cluster cutting method. Only units with clear boundaries and $<0.5 \%$ of spike intervals within a $3 \mathrm{~ms}$ refractory period are included in the present analysis. Typically we isolated one or two distinct neurons from each microwire, but in several cases, we observed up to four distinct neurons from a single microwire. The instantaneous spike frequency was measured by convolving the timing of each unit with a Gaussian function of an SD of $20 \mathrm{~ms}$.

Phase locking between spikes and field oscillations. To analyze the phase locking between single units and field oscillations, we used a recently published procedure (Jacobs et al., 2007). We first downsampled recordings to $2 \mathrm{kHz}$. To minimize the contribution of low-frequency components of spikes toward spectral calculations, the samples from $2 \mathrm{~ms}$ before to $8 \mathrm{~ms}$ after each spike were replaced with a linear interpolation of the underlying field signal. Then, we computed oscillatory phase and power of the field potential using Morlet wavelets at frequencies between 30 and $240 \mathrm{~Hz}$. We considered a neuron phase locked at a particular frequency if the hypothesis of circular uniformity for its field phase distribution could be rejected at $p<0.001$ using a Bonferroni-corrected Rayleigh test (Fisher, 1993) (see supplemental material, available at www.jneurosci.org). 
A

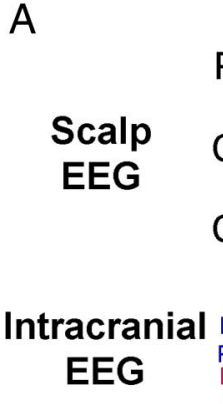

$\mathrm{B}$
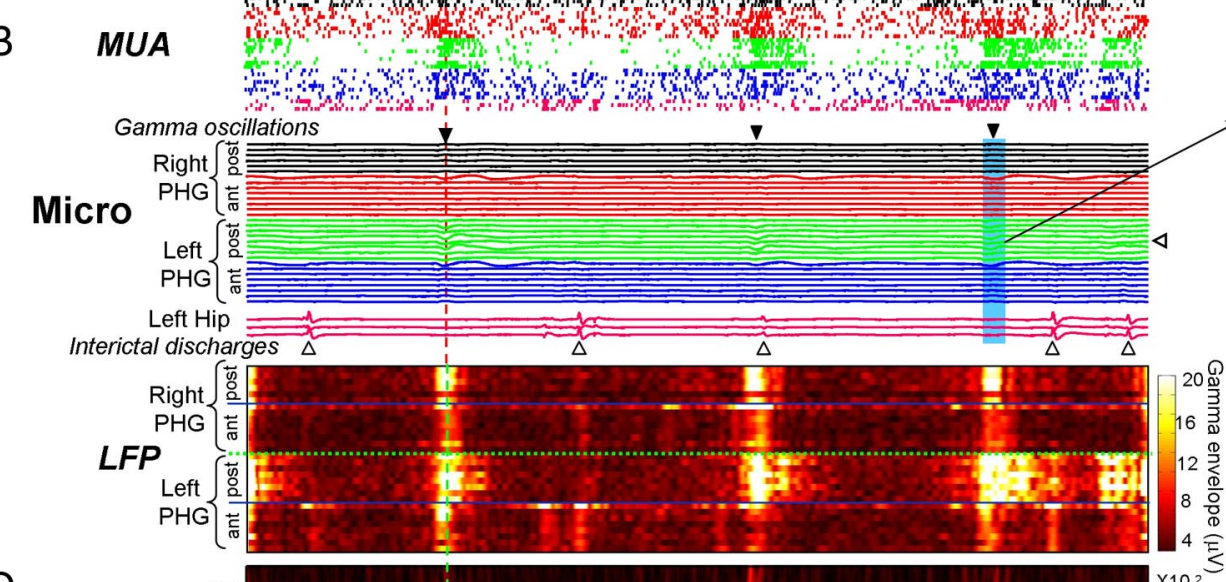

D

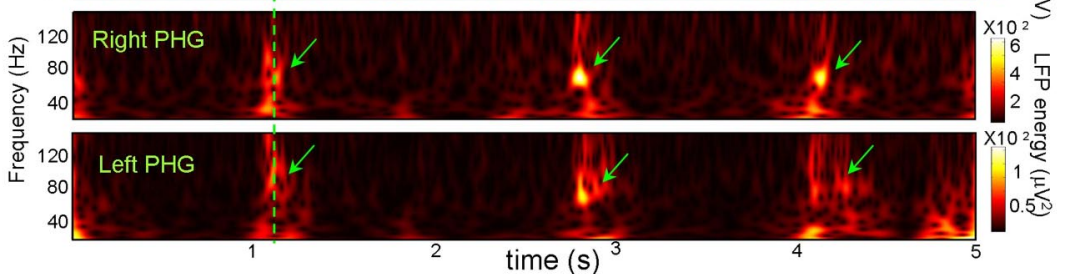

C Right PHG - Filtered $(40-120 \mathrm{~Hz})$

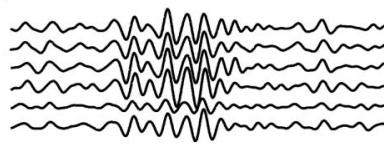

Right PHG - broad band
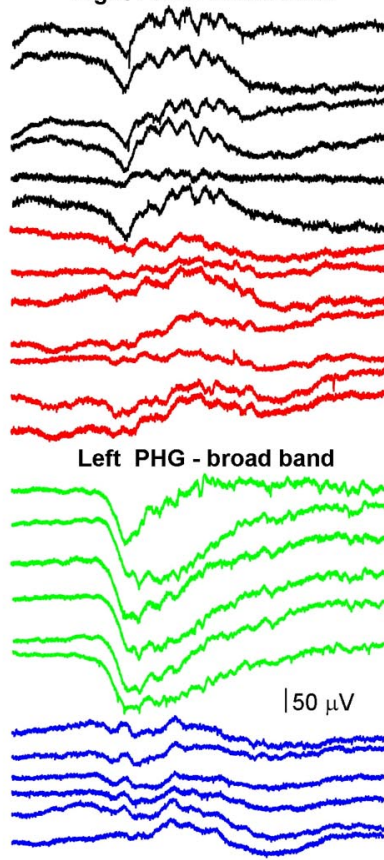

Left Hip - broad band

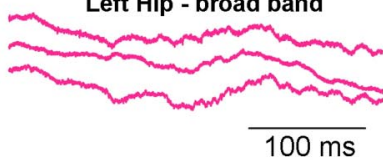

Figure 1. Gamma patterns recorded during sleep stage 3. A, Slow waves, fulfilling standard polysomnographic criteria of duration $(a)$ and amplitude $(b$, $c$; see text) were recorded from several scalp EEG channels (here C3, C4, and Pz following the 10-20 international system of electrode placement, subject 1). B, Gamma episodes (black triangles) simultaneously recorded across 30 microelectrodes (Micro) in the right and left parahippocampal gyri (PHG; ant, anterior part; post, posterior part). One channel (white triangle on right side of traces) was selected for oscillation detections. In most of the cases, these oscillations were not associated with interictal discharges recorded with microelectrodes (white triangles) or intracranial EEG electrodes $(\boldsymbol{A})$ in the left epileptogenic hippocampus (Hip). Bottom, Envelope amplitudes of the filtered LFPs in the gamma range. Note that gamma activities formed large spatial patterns occurring almost simultaneously between all recorded cortical sites and were temporally correlated with positive peaks (i.e., up deviations) of EEG slow waves (asterisks in $A$ ). Strong MUAs were observed during gamma episodes. C, Display of a single gamma episode appearing simultaneously, in either the raw signals or those filtered between $40 \mathrm{and} 120 \mathrm{~Hz}$ (top), in the right and left posterior parahippocampal gyri. $D$, Corresponding wavelet transforms of two homotopic sites revealing nearly simultaneous gamma oscillations with distinct narrow band frequencies around $70-80 \mathrm{~Hz}$ (green arrows).

Spike synchronization. A statistical strategy was used to assess temporal structure in spike trains (Hatsopoulos et al., 2003). Each spike in each neuron in the original dataset was randomly and independently perturbed (or "jittered") on a uniform interval of $[-2.5,+2.5] \mathrm{ms}$ to form a surrogate dataset. By repeating the procedure 1000 times, the $99.9 \%$ confidence intervals for each bin $(p=0.001)$ is calculated. Coincident firings were determined to be statistically significant if the original crosscorrelogram deviated from cross-correlogram constructed from the jittered datasets.

\section{Results}

\section{Gamma oscillations during UP states in SWS}

Episodes of gamma activity were automatically identified using previous methodology (Staba et al., 2004) and were visually confirmed. Figure $1 B$ illustrates typical patterns of detected gamma activities recorded across 30 microelectrodes in the right and left posterior parahippocampal gyri (subject 1 , sleep stage 3 ). In either the raw signals or those filtered between 40 and $120 \mathrm{~Hz}$, large-amplitude fast sinusoidal waves appeared in many channels as discrete events that were clearly distinguishable from background activity (Fig. 1C). As best seen in the envelope amplitude of the filtered signals in the gamma range (Fig. $1 B$, bottom), these occurrences of gamma activity appeared simultaneously between homotopic sites, forming large spatiotemporally coherent patterns of increased activity separated by equally coherent periods of little activity. The wavelet transformed-energy scalogram, which represents the spectral energy with respect to time and frequency, more clearly illustrates gamma activity in homotopic parahippocampal sites between hemispheres (Fig. 1D). Prominent gamma frequency oscillations, with distinct narrow band peaks centered around $70-80 \mathrm{~Hz}$, were observed lasting a few hundred milliseconds. These oscillations were recorded from a patient with mesial temporal lobe epilepsy who had seizures starting from the left hippocampal formation. In this patient, short interictal discharges (epileptic spikes or sharp waves), characterized by high amplitude $(>50 \mu \mathrm{V})$ and $<200 \mathrm{~ms}$ duration, could simultaneously be identified on both intracranial EEG and nearby microelectrode recordings of the hippocampus (Fig. $1 A, B)$. As seen in a large percentage of cases $(88 \%)$, and also in other patients with mesial temporal lobe epilepsy $(n=3$ subjects with simultaneous intracranial EEG and microwires recordings), 
A

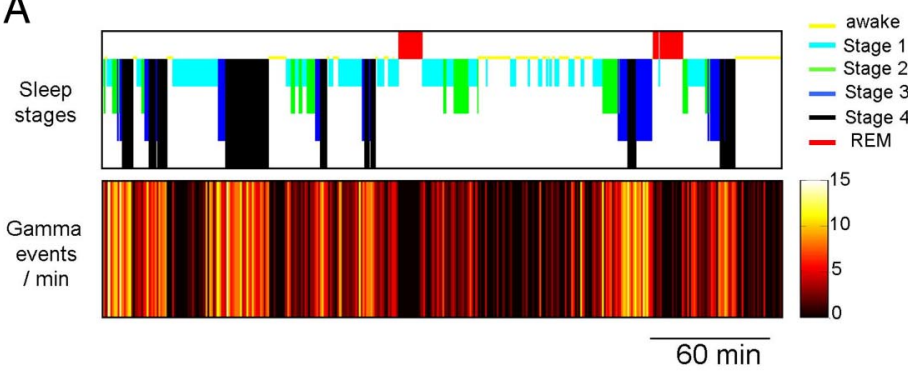

B

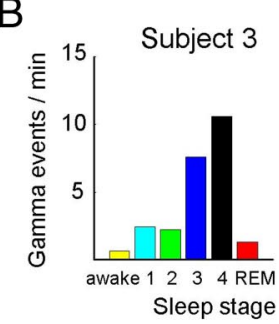

D awake

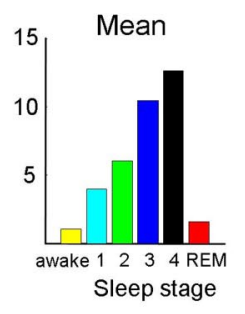

C

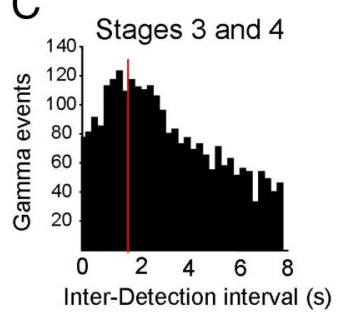

stage 2
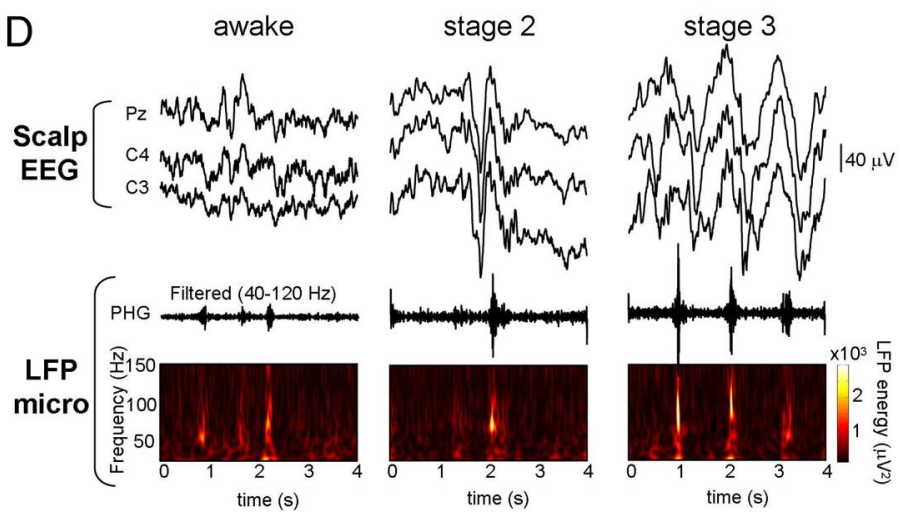
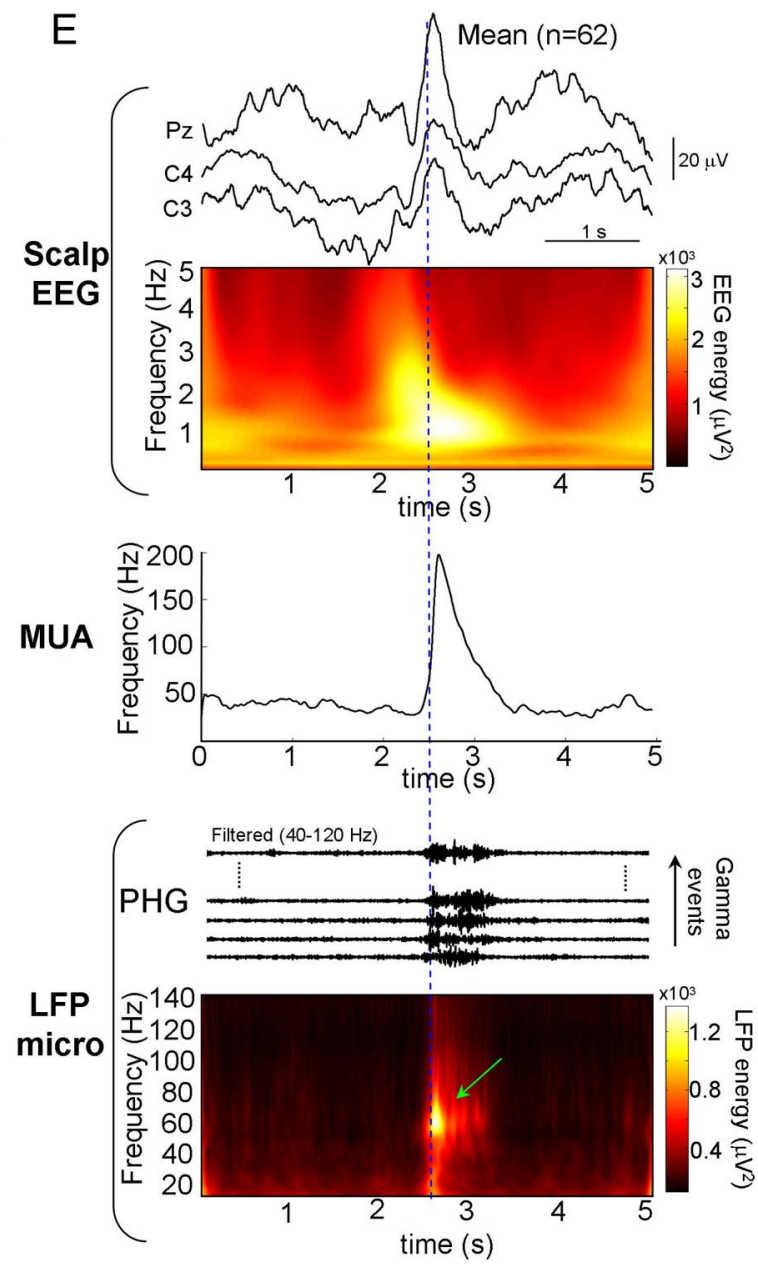

Figure 2. Gamma and scalp EEG patterns during polysomnographically defined sleep-wake states. A, All-night detections of gamma patterns (here recorded in the posterior parahippocampal gyrus, subject 3) during all physiological stages from quiet wakefulness, sleep stage 1- 4 and REM. The number of detected gamma patterns per minute was indicated in colors a function of sleep stages. Note the increased occurrence of slow oscillations as sleep deepens. $\boldsymbol{B}$, Mean number of gamma patterns per minute as a function of sleep stages for one subject and in average over six subjects. C, Distribution of interdetection intervals measured during sleep stages 3 and 4 . The distribution peaks at $1.7 \mathrm{~s}$ (red line), indicating that the main frequency of gamma oscillation occurrence during deep sleep was $\sim 0.6 \mathrm{~Hz}$. D, Gamma oscillations can appear during wakefulness without clear EEG waves in scalp EEG but have a tendency to emerge most frequently and with higher amplitude during SWS and were temporally correlated with positive peaks of EEG slow waves (subject 1, electrodes C3, C4, and Pz following the 10-20 international system). E, Bottom, Gamma events during SWS were aligned on their initiation and the mean wavelet transforms of one LFP channel is illustrated, showing gamma-frequency components (green arrow). Middle, Gamma oscillation start-time-triggered frequencies of MUAs. Top, Gamma oscillation start-time-triggered EEG averages. The mean wavelet transforms of one EEG channel (Pz) is depicted, showing low-frequency components in the averaged signals. Note that the gamma events were correlated with positive slow waves of scalp EEG and an increase in the frequency of MUAs (subject 1).

gamma oscillations in the parahippocampal gyri were not coincident with these discharges (within a time window of $200 \mathrm{~ms}$ ) (Fig. 1C), suggesting that they can arise independently from synchronous activities of the epileptogenic hippocampus.

Time series analysis of gamma episodes in relation to the sleep-wake cycle revealed that gamma was present across all stages of vigilance (Fig. 2A). Gamma was less frequent during wakefulness, stage 1, and REM (Fig. $2 B$ ), and more prominent during stage 2 , and high rates occurred during sleep stages 3 and 4. Across six subjects, the mean number $( \pm S D)$ of gamma episodes recorded in the parahippocampal gyrus was $13.3 \pm 6.4$ per minute in sleep stages 3 and 4. During these deeper stages of sleep, the gamma patterns often emerged in rhythmical sequences (for examples, see Figs. $1 A, 2 D$ ) with a main interepisode interval of $1.7 \pm 0.5 \mathrm{~s}$ ( $n=6$ subjects) (Fig. $2 C)$, corresponding to a frequency of $\sim 0.6 \mathrm{~Hz}$, suggesting that they were associated with macroscopic slow-wave oscillations.

Analysis of scalp EEG during SWS showed that local gamma oscillations at deep sites were temporally correlated with the surface-positive components of large-amplitude slow waves. Figure $1 A$ shows a typical example of these slow waves during stage 3. Over four subjects, gamma oscillation onset-triggered EEG averages confirmed that gamma bursts were associated with the surface-positive peak of slow waves (Fig. 2 E). We asked whether these waves represent genuine sleep slow waves (SW). First, most of these waves ( $\sim 80 \%$ in 4 patients) fulfilled the following standard criteria for SW (Massimini et al., 2004) (see Fig. $1 \mathrm{~A}$ and supplemental Fig. 1, available at www.jneurosci.org as supplemental material): (1) a negative-slope zero crossing and a subsequent positive-slope zero crossing separated by at least $300 \mathrm{~ms}$ (in average over all detected events: $a=487 \pm 120 \mathrm{~ms}$ at $\mathrm{Pz}$ ), (2) a negative peak between the two zero crossings with voltage $<80$ $\mu \mathrm{V}(b=100 \pm 37 \mu \mathrm{V})$, and (3) a negative-to-positive peak-topeak amplitude $>140 \mu \mathrm{V}(c=170 \pm 45 \mu \mathrm{V})$. Second, during stage 2 , the corresponding surface-positive portion of the wave was often associated with spindles at $\sim 7-14 \mathrm{~Hz}$, suggesting that they were K-complexes (Amzica and Steriade, 1997) (supplemental Fig. 1, available at www.jneurosci.org as supplemental 

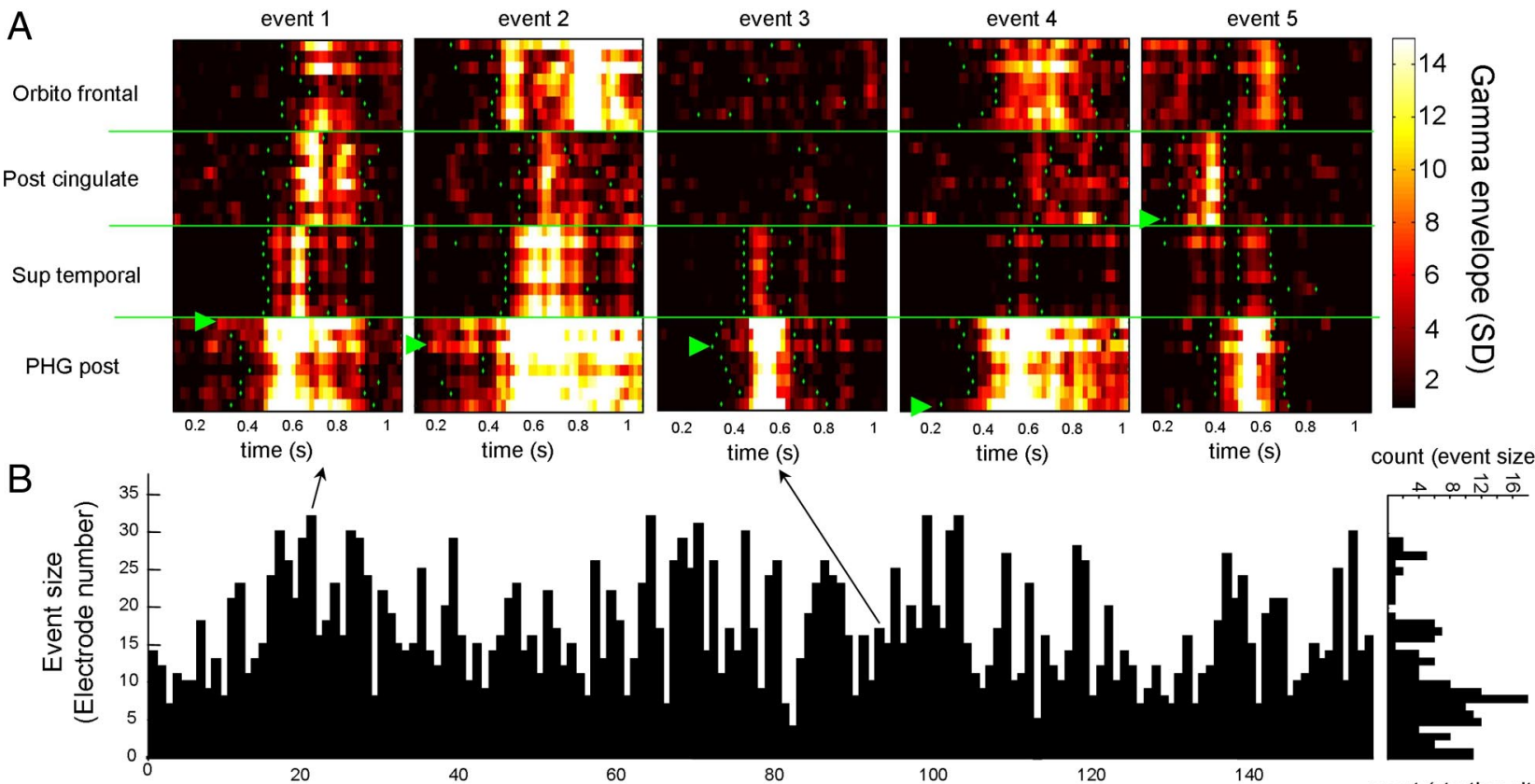

$\uparrow$

time (s)

(s)

count (event size)

C

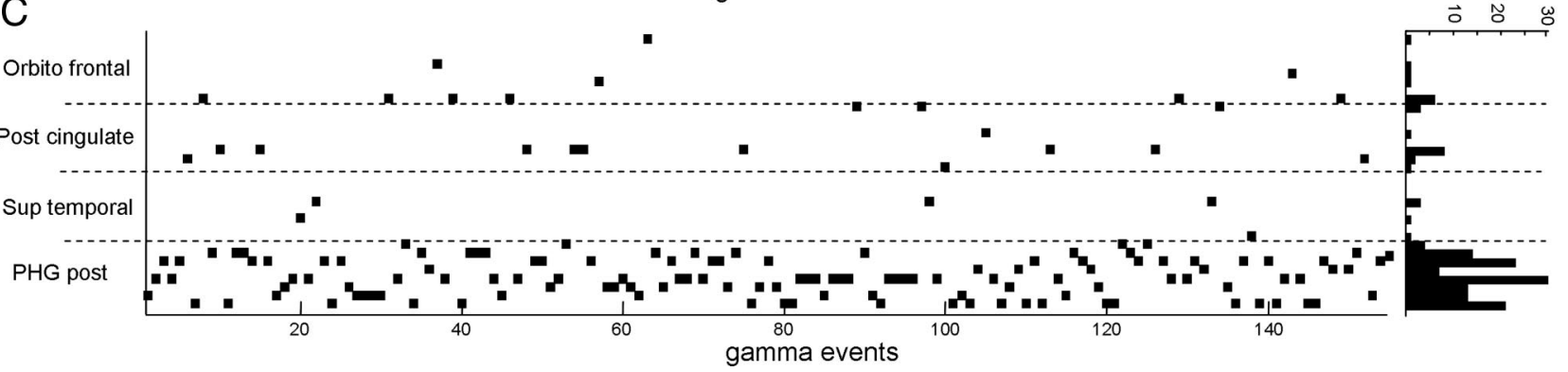

Figure 3. Spatial distribution of gamma patterns. $\boldsymbol{A}$, Examples of spatial distribution of 5 individual gamma patterns (subject 2 ). Note the strong variability of involved electrodes and location of the starting site (green triangle). $\boldsymbol{B}$, Number of active microelectrodes for each gamma pattern detected over the all-night recording session of the same subject. Right histogram, Distribution of event sizes as a function of the number of electrodes expressing them. C, Location of starting sites for each gamma patterns detected over the entire recording session. Right histogram, Distribution of starting locations.

material). Third, for patients with mesial temporal lobe epilepsy $(n=4)$, simultaneous recordings of the epileptogenic hippocampus [for example, the left hippocampus in subject 1 (see Fig. 1)] revealed that scalp slow components were not reflecting epileptic spikes emerging from the epileptogenic zone and spreading to distant nonepileptic regions. In particular, epileptic spikes had markedly different temporal dynamics with extremely fast transients $(<70 \mathrm{~ms})$. Finally, as demonstrated by animal studies (Steriade et al., 1993), a general increase in cellular discharges is associated with the surface-positive portion of SW, referred as UP state. Consistent with another recent human study using microelectrode and macroelectrode intracranial arrays (Cash et al., 2009), simultaneous recordings of MUA confirmed that a significant number of neurons increased their rate of discharge during superficial positive slow components (Figs. $1 A, B, 2 E$ ). In summary, our observations suggest that gamma oscillations during SWS were reliably associated with normal UP states.

\section{Spatial components of SWS gamma oscillations}

During SWS, gamma episodes were observed in all investigated cortical areas (9/9 subjects). While gamma episodes were recorded in a variety of cortical locations (superior temporal gyrus: $16 / 16$ channels in $2 / 2$ subjects, posterior cingulate cortex: $8 / 8$ in $1 / 1$ subjects, the supplementary motor area: $8 / 8$ channels in $1 / 1$ subjects), the strongest rates of detection and the highest amplitudes in gamma power occurred in the posterior parahippocampal gyrus (90/136 channels found in 8/8 subjects; $40 \pm 35 \mu \mathrm{V}$ peak-to-peak amplitude) and entorhinal cortex (32/48 in $3 / 4$ subjects; $21 \pm 13 \mu \mathrm{V})$. Less frequently and of smaller amplitude, gamma activities were detected in orbitofrontal cortex $(8 / 16$ channels in $1 / 2$ subjects), anterior cingulate cortex (8/52 in $1 / 4$ subjects), and occipital cortex (8/32 channels in $1 / 2$ subjects).

To further investigate the spatial distribution of gamma patterns, we quantitatively examined how gamma episodes fluctuated in both space and time. For each discrete gamma incidence during SWS, we recorded the number and location of the channels where the gamma power attained a value over a given threshold ( $>3$ SD from baseline mean). We defined the number of active microelectrodes and the duration during which they remained above threshold. In four of nine subjects, gamma episodes (lasting for an average period of $580 \pm 88 \mathrm{~ms}$ ) remained local and involved all eight channels within a single cortical region (the parahippocampal gyrus in three of four subjects; and the entorhinal cortex in one of four subjects) without affecting other sites. In five of nine subjects, gamma episodes had a more complex spatiotemporal distribution and often occurred in multiple cortical areas at approximately the same time (see individual events in Fig. $3 A$ ). Figure $3 B$ plots the number of active micro- 
A
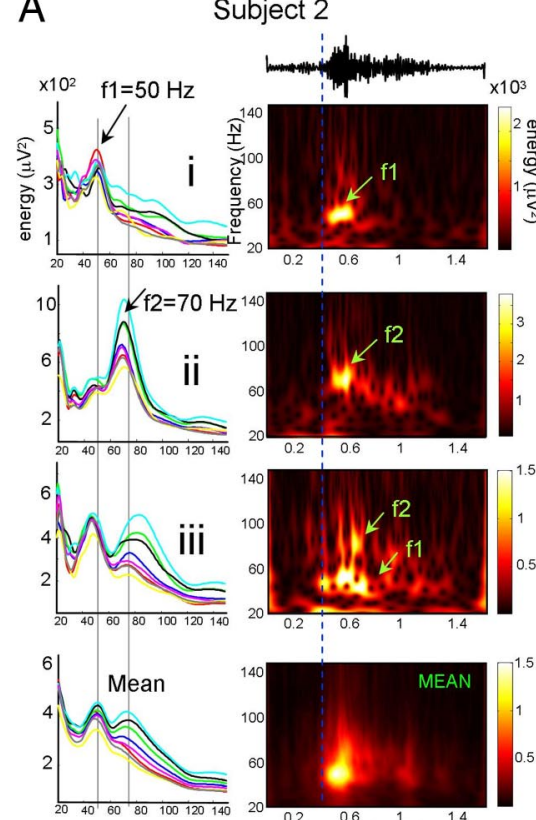

Frequency $(\mathrm{Hz})$
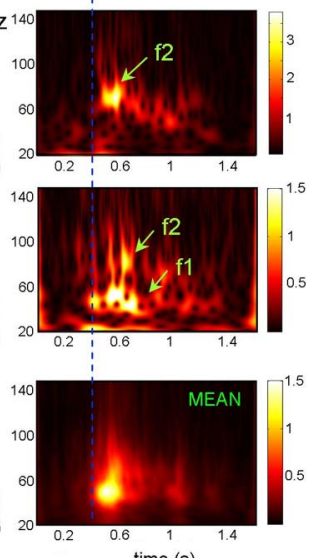

D

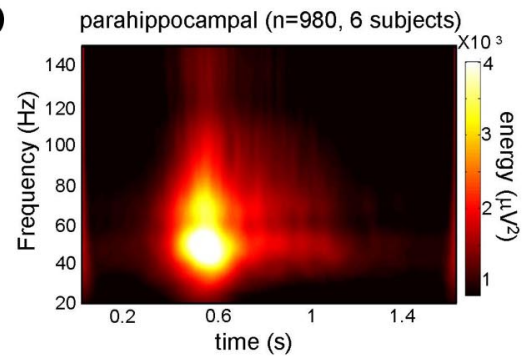

B
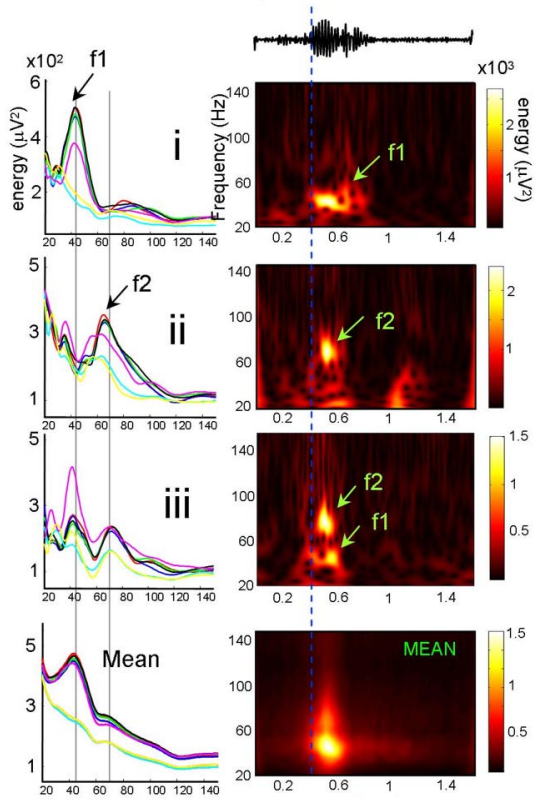

Frequency $(\mathrm{Hz})$

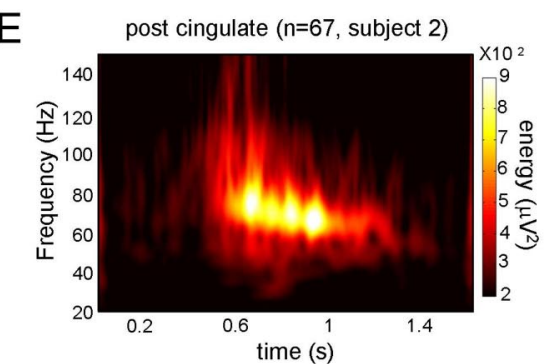

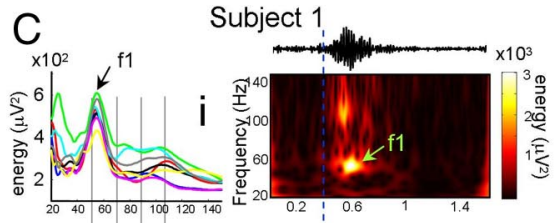
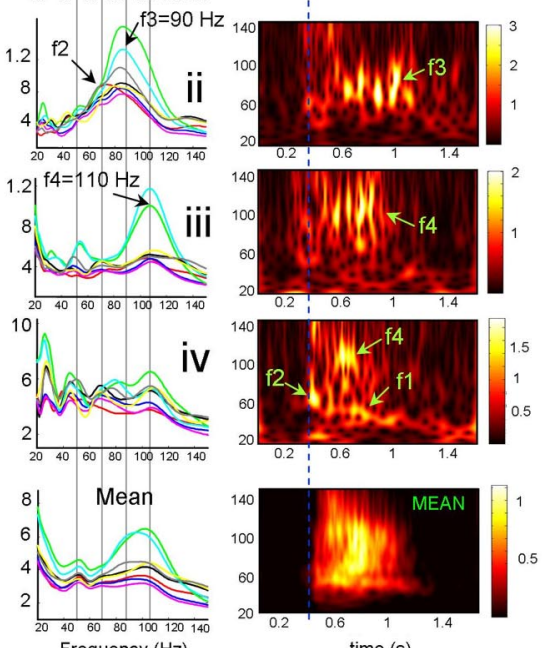

$\mathrm{F}$

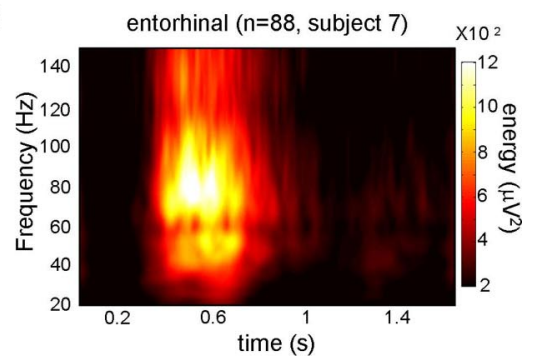

Figure 4. Frequency components of gamma oscillations. $\boldsymbol{A}-\boldsymbol{C}$, The power spectra of individual gamma oscillations (i-iii) and global means (bottom) recorded in the parahippocampal gyrus of three subjects. The spectra of eight individual channels are depicted by different colors in the left panels. The time-frequency maps of one particular channel are illustrated in the right panels. $\boldsymbol{D}-\boldsymbol{F}$, Average time-frequency maps of gamma events in the parahippocampal gyri of six subjects $(\boldsymbol{D})$, posterior cingulate cortex of one subject $(\boldsymbol{E})$, and entorhinal cortex of another subject $(\boldsymbol{F})$.

electrodes for each gamma event detected across the entire recording session of one subject. Frequently (in $70 \%$ of cases), these patterns involved only a single local cortical area or extended to only one adjacent area ( $<16$ channels) (see Fig. $3 A$, event 3 ). Occasionally (in $30 \%$ of cases), these patterns involved a large number of channels (ranging from 16 to 32 ) (see Fig. 3A, events 1 , 2,5 ), with a broad distribution of events across all microelectrodes. The duration of these events was highly variable (range $280-820 \mathrm{~ms}$ with $681 \pm 210 \mathrm{~ms}$ on average in five subjects) (for examples, see Fig. $3 A$, events 1,2 ).

For each gamma episode, we also evaluated the microelectrode at which it was recorded first to determine whether gamma activity originated predominantly at specific locations. For one subject, Figure $3 C$ plots the earliest activations for each gamma pattern detected across the all-night recording session. As also observed in four of five subjects, although gamma patterns could occasionally appear at multiple locations (e.g., cingulate cortex, orbitofrontal cortex, superior temporal cortex) (see Fig. $3 A$, event 5), a hot spot of early activation was centered in the parahippocampal gyrus. These large-scale gamma patterns often occurred bilaterally and could originate in either hemisphere (supplemental Fig. 2C, available at www.jneurosci.org as supplemental material). For patients with mesial temporal lobe epilepsy $(n=4)$, visual assessment revealed that large-scale gamma patterns were not coincident (within a time window of
$200 \mathrm{~ms}$ ) with interictal spikes emerging from the epileptic hippocampal formations and spreading to lateral or contralateral brain regions (Fig. 1).

\section{Frequency components of SWS gamma oscillations}

Our next step was to characterize the frequency components of SWS gamma activities using continuous wavelet transform. For our group analysis, we focused on patterns occurring in the parahippocampal gyrus (Fig. 4A-D). On average over six subjects, quantitative analysis revealed that a broad range of activity from 40 to $120 \mathrm{~Hz}$ increased by $>300 \%$ compared to control periods of $0.5 \mathrm{~s}$ before gamma oscillations (Fig. $4 D$ ). As well, when looking at individual subjects and single detected events (Fig. 4A-C), it was apparent that gamma activity often corresponds to discrete oscillatory bursts appearing in narrow frequency bands. In six of six subjects, two main power peaks can be seen in the low-gamma range around $f_{1}=40-50 \mathrm{~Hz}$ and $f_{2}=70-80 \mathrm{~Hz}$. These oscillations could appear independently at overlapping locations (detection rates of $21 \%$ for $f_{1}$ and $17 \%$ for $f_{2}$ over all events) (see individual events $i$ and $i$ in Fig. $4 A, B$ ) but most of the time emerged together in mixed spatiotemporal patterns (detection rate of $62 \%$ ) (see individual event iii in Fig. $4 A, B$ ). This latter pattern was associated with, on average, a broad band activity between 40 and $80 \mathrm{~Hz}$ (means in Fig. 4A,B). In all cases, these low-gamma oscillations involved all eight channels over the para- 

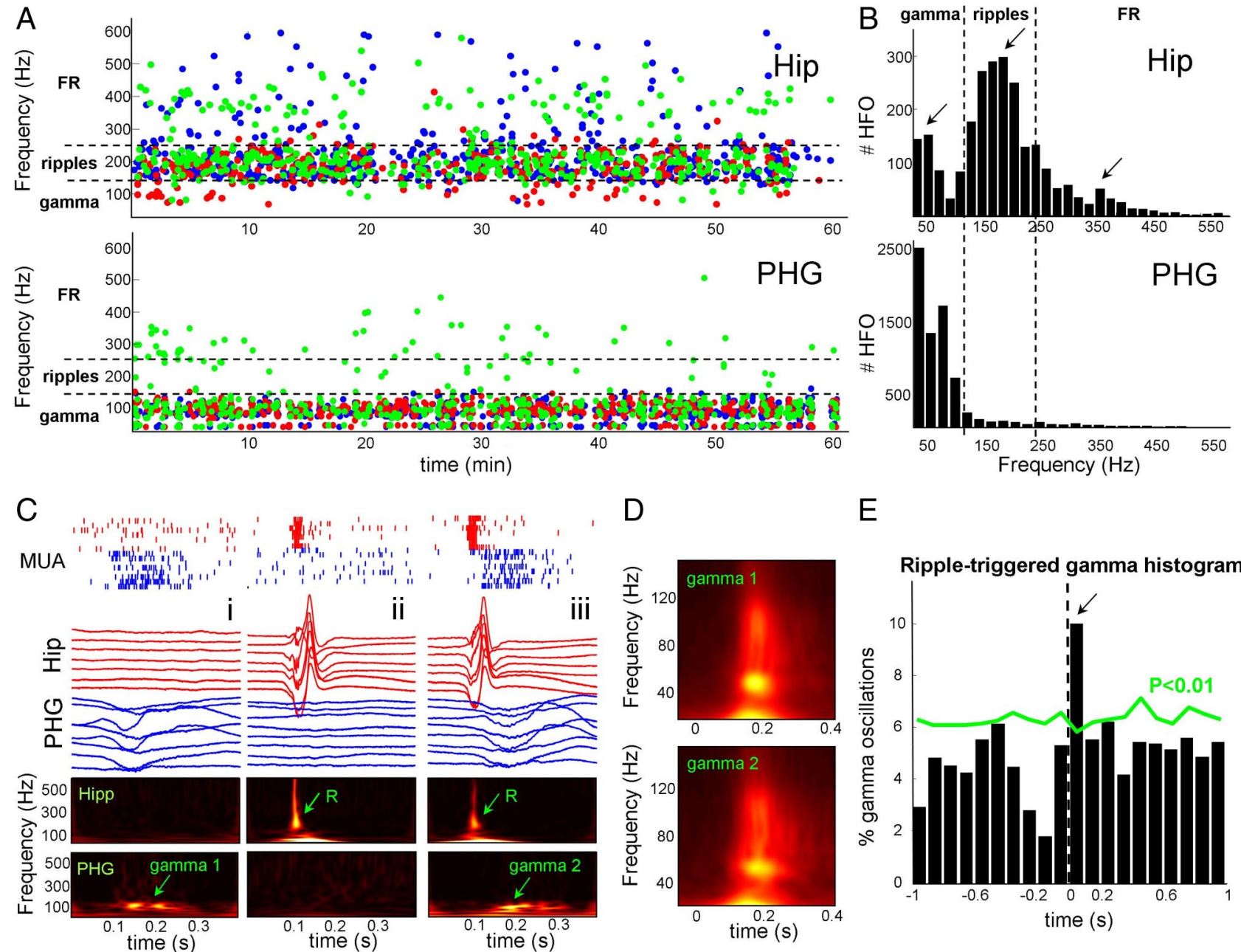

$\mathrm{D}$

E
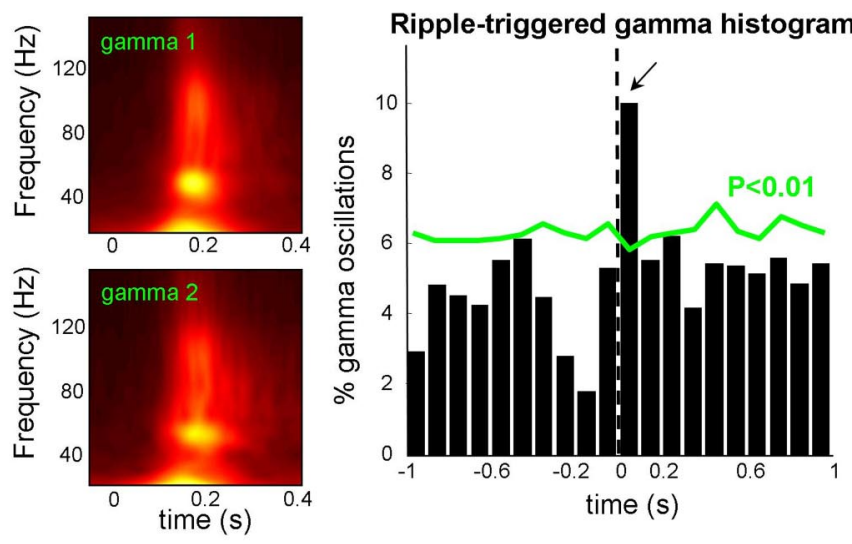

Figure 5. High-frequency oscillations in the parahippocampal cortex and hippocampus. $\boldsymbol{A}$, Detection times and mean frequencies of high-frequency oscillations (gamma, ripples, and FR) between 40 and $600 \mathrm{~Hz}$ (three individual channels are depicted by different colors) for a single subject (subject 1). $\boldsymbol{B}$, Frequency histogram over five subjects in the hippocampus (Hip) and adjacent parahippocampal gyrus (PHG). Note, in the hippocampus, a multimodal distribution in the high-frequency range with three main peaks in the gamma, ripples, and fast ripple range. In contrast, in the parahippocampal gyrus, only one single-frequency band was dominant in the gamma range between 40 and $130 \mathrm{~Hz}$. C, In individual events $i$ and $i$, hippocampal ripple/sharp wave complexes and parahippocampal gamma oscillations (gamma 1) were not coincident within a time window of $100 \mathrm{~ms}$. Nevertheless, in event iii, parahippocampal gamma oscillations (gamma 2) immediately followed hippocampal ripple/sharp wave complexes. $\boldsymbol{D}$. Average time-frequency maps of gamma events not following $(n=115$ events, gamma 1$)$ or following $(n=43$ events, gamma 2$)$ ripple/sharp waves in three subjects. $E$, Ripple onset-triggered histograms of gamma oscillations ( $n=288$ ripples in 5 subjects).

hippocampal gyrus (Fig. $4 A, B$, left). In two of six subjects, in addition to low-gamma oscillations, two other peaks could be detected in the high-gamma range around $f_{3}=90-100 \mathrm{~Hz}$ and $f_{4}=100-120 \mathrm{~Hz}$. These high-gamma oscillations could appear independently at discrete locations involving two to three channels (detection rates of $31 \%$ for $f_{3}$ and $24 \%$ for $f_{4}$ ) (see individual events $i$ and $i i i$ in Fig. $4 C$ ) but, most frequently, emerged together with low-gamma oscillations (detection rate of $45 \%$ ) (individual event iv in Fig. 4C; see also supplemental Fig. 3, available at www. jneurosci.org as supplemental material), giving on average the appearance of a broad band spectrum between 40 and $120 \mathrm{~Hz}$ (mean in Fig. 4C). Similar narrow band oscillations at low- and high-gamma frequencies also occurred in other cortical locations (e.g., entorhinal cortex, posterior cingulate cortex, orbitofrontal cortex, and superior temporal cortex) (see Fig. $4 E, F$ ).

High-frequency oscillations in the parahippocampal cortex and hippocampus

Ripples $(100-250 \mathrm{~Hz})$ and fast ripples $(\mathrm{FR}, 250-500 \mathrm{~Hz})$ have been previously identified with microelectrodes in human hip- pocampus (Bragin et al., 1999, 2002) and were more prominent during non-REM sleep (Staba et al., 2004). FR occurrences are significantly associated with seizure-generating areas (Bragin et al., 2002; Engel et al., 2009). For investigating differences between these fast oscillations and gamma, we have systematically detected during SWS, ipsilateral to side of seizure onset, all highfrequency oscillations between 40 and $600 \mathrm{~Hz}$ in the hippocampus and adjacent parahippocampal gyri of five subjects. In the hippocampus, analysis of frequencies at maximum power showed a multimodal distribution in the high-frequency range with three main peaks around 50, 190, and $360 \mathrm{~Hz}$ (occurrence times for a single subject in Fig. 5A, and histogram over all subjects in Fig. 5B). On the basis of these data, and results from our previous studies, subsequent analyses of gamma, ripples, and FR were based on the following classification: events between 40 and $130 \mathrm{~Hz}$ were labeled gamma oscillations, whereas events within the frequency bands of $130-250 \mathrm{~Hz}$ and $250-600 \mathrm{~Hz}$ were labeled ripples and FR, respectively. As previously reported, both ripples and FR were strongly expressed in the epileptogenic hippocampus during SWS (ripple rate of $2.8 \pm 2.3$ per minute; FR 
rate of $1.3 \pm 1.1$ per minute). Ripples and FR could appear independently at a few discrete locations and, in a large percentage of cases $(\sim 85 \%)$, were associated with sharp waves. At a very low rate $(0.27 \pm 0.12$ per minute), oscillations in the gamma range can also be identified in the hippocampus of all investigated subjects. These gamma oscillations were dominant in the low frequency range with a main peak around $50 \mathrm{~Hz}$ and possessed larger spatial profiles than ripples (supplemental Fig. 4, available at www.jneurosci.org as supplemental material), suggesting that they were generated by different neuronal sources. In contrast, in the parahippocampal gyri of all investigated subjects, only one single-frequency band was dominant in the gamma range between 40 and $130 \mathrm{~Hz}$ (Fig. $5 A, B$ ). In two of five cases (subjects 1 and 2), consistent with the spatial extent of seizure onset zone (supplemental Table 1, available at www.jneurosci.org as supplemental material), ripples and FR occurred at a low rate $(1.13 \pm$ 0.58 per minute) at discrete locations involving one to two channels (for an example, see Fig. 5A) and were often temporally correlated with the occurrences of gamma oscillations (supplemental Fig. 4, available at www.jneurosci.org as supplemental material). The absence of FR in three of five subjects suggests that the investigated parahippocampal gyri were not involved in primary epileptogenic regions, suggesting that the reported gamma oscillations are not a product of pathological hypersynchronization. Our final step was to examine time relations between hippocampal ripples/sharp wave complexes and parahippocampal gamma oscillations. On the basis of their oscillation frequency $(130-250 \mathrm{~Hz})$ and wave duration $(70-200 \mathrm{~ms})$, we visually isolated ripples/sharp wave complexes in the hippocampus $(n=288$ in 5 subjects) and examined their temporal correlation with gamma oscillations in the adjacent parahippocampal gyrus. As described previously on the basis of their underlying cellular firing patterns (Le Van Quyen et al., 2008), these ripple oscillations can be considered normal electrographic phenomena in the hippocampus. Most of the time ( $\sim 90 \%)$, hippocampal ripples and parahippocampal gamma oscillations were not coincident within a time window of $100 \mathrm{~ms}$, suggesting they were independent phenomena (Fig. 5C, examples $i$ and $i$ ). Nevertheless, in a significant number of the cases $(\sim 10 \%)$, we found that parahippocampal gamma oscillations immediately followed hippocampal ripple/ sharp wave complexes (Fig. 5C, example iii). No significant difference was found in magnitude, frequency, or duration between gamma oscillations following ripple/sharp wave complexes and gamma in the absence of ripple/sharp waves ( $n=3$ subjects) (Fig. $5 D$ ). Ripple onset-triggered histograms of gamma oscillations confirmed that, in a statistical sense, the number of parahippocampal gamma oscillations within a time window of $100 \mathrm{~ms}$ after hippocampal ripple/sharp wave complexes was greater than chance (i.e., exceeded the 99th percentile of the distribution, computed 100 times from randomly and independently shuffled ripples, $p<0.01$ ) (Fig. 5E). This suggests that at least a fraction of the ripples/sharp waves were coupled with gamma oscillations and these coincidences may play a coordinating role in the hippocampo-neocortical interactions during SWS.

\section{Short-range intracortical coherence of SWS gamma oscillations}

Although gamma episodes frequently occurred almost simultaneously between distinct cortical sites during SWS (see Fig. 1), cross-correlogram functions suggested that the spatial extent of cortical foci oscillating in phase was only local (Fig. 6). Locally, cross-correlograms between filtered $(40-120 \mathrm{~Hz})$ traces revealed that gamma activities are strongly phase locked in a majority of case (95\%) with near zero phase lag (time lags of $0-3 \mathrm{~ms}$ ) between different sites separated by $\sim 500 \mu \mathrm{m}$ over the same cortical area (see Fig. 6A, cross-correlogram 1-2 between two microelectrodes within the right posterior parahippocampal gyrus). As well, no phase reversal of gamma could be detected on multiple recordings of single cortical regions (supplemental Fig. 5, available at www.jneurosci.org as supplemental material). Since we used multichannel bundles of microelectrodes randomly spanning different cortical layers, this suggests that gamma oscillations are in phase across the cortical depth. On a larger spatial scale, phase locking fell off quickly when computed between different adjacent neocortical sites [see Fig. 6A, cross-correlogram between microelectrode 1 (right parahippocampal gyrus, posterior part) and 3 (right parahippocampal gyrus, anterior part)]. No reliable differences in short- or long-distance coherence were observed between low- and high-gamma frequency bands. Similar results were found in five of five subjects exhibiting large-scale spatial patterns of gamma activity (see Fig. $6 C$ for the averaged results in three subjects). Although gamma generally occurred almost simultaneously between the homotopic sites, there was no strict phase correlation across the hemispheres [see Fig. 6A, crosscorrelogram between 1 (right posterior parahippocampal gyrus) and 4 (left posterior parahippocampal gyrus)]. Occasionally (in two of five subjects), coherent gamma oscillations were observed between adjacent cortical areas over short distances $(<10 \mathrm{~mm})$ [see Fig. $6 B$, cross-correlogram between 1 (right posterior parahippocampal gyrus) and 3 (right superior temporal cortex), Fig. 6C for averaged results; see also supplemental Fig. 6 (available at www.jneurosci.org as supplemental material) for details].

\section{Gamma oscillation-related discharge of neurons}

Consistent with a switch to the UP state, recordings of multiunit activity indicate that a significant number of neurons increased their discharge frequency during occurrence of gamma oscillations in SWS (Figs. 1A, 2E, 7A). To gain further insight into single-unit discharges, we examined the discharge characteristics of putative single neurons (supplemental Fig. 7, available at www. jneurosci.org as supplemental material). A total of 206 single cells were detected and selected for analysis in seven subjects $(n=93$ in the parahippocampal gyrus; $n=35$ in the posterior and anterior cingulate cortex; $n=49$ in the entorhinal cortex; $n=17$ in the orbitofrontal cortex; $n=8$ in the superior temporal cortex; $n=4$ in the supplementary motor area). For the cells in the parahippocampal gyrus, the mean firing rate was $3.54 \pm 2.19$ spikes per second, the mean burst rate was $9.62 \pm 4.4$ bursts per minute and most of the investigated cells (73\%) fall within the nonepileptic category (Staba et al., 2002). When gamma episodes were detected on a microelectrode, the majority of corresponding single neurons $(70 \%)$ exhibited a significant increase in spike discharges compared to control periods of $0.5 \mathrm{~s}$ before gamma oscillations (Fig. 7B, top, an example of 23 simultaneously recorded cells) with a large proportion (21\%) firing at high frequency between 20 and $40 \mathrm{~Hz}$ (Fig. 7B, bottom). Nevertheless, during a given gamma event, individual neurons participate sparsely in the population rhythm: cell discharges do not typically show a clear rhythm, but their timing is significantly phase locked to the population oscillations.

To quantitatively analyze the phase relationships between unit discharges and local gamma activity, we evaluated the phase values of spikes relative to the field using a wavelet decomposition (Jacobs et al., 2007; Le Van Quyen et al., 2008). We found that $33 \%$ of the cells were significantly phase locked to the local gamma oscillations $(p=0.01)$. Figure $7 C i$ illustrates the phase 
A
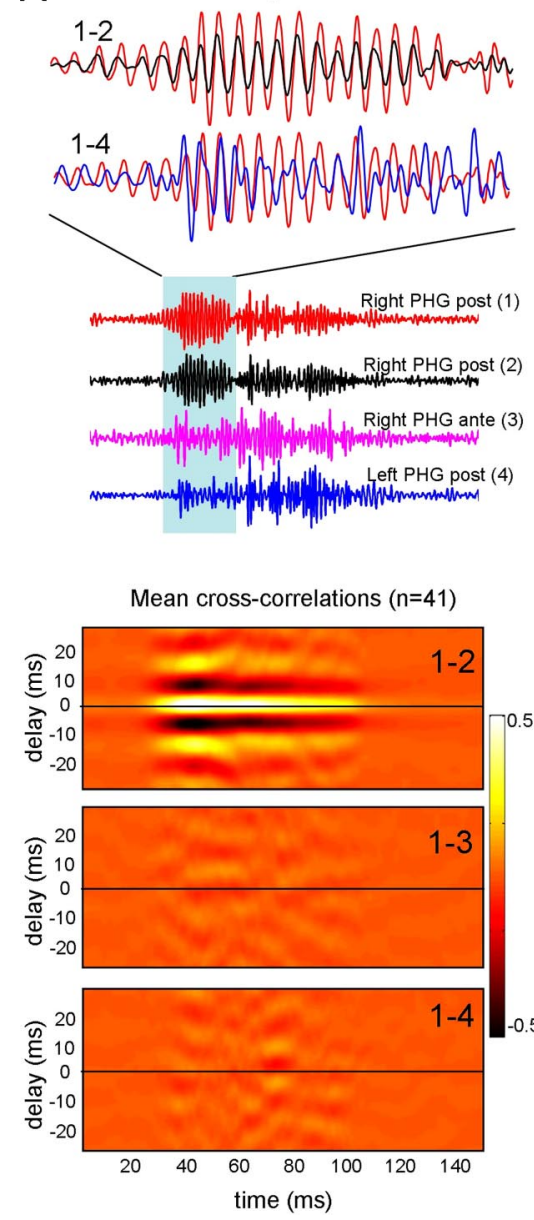

B
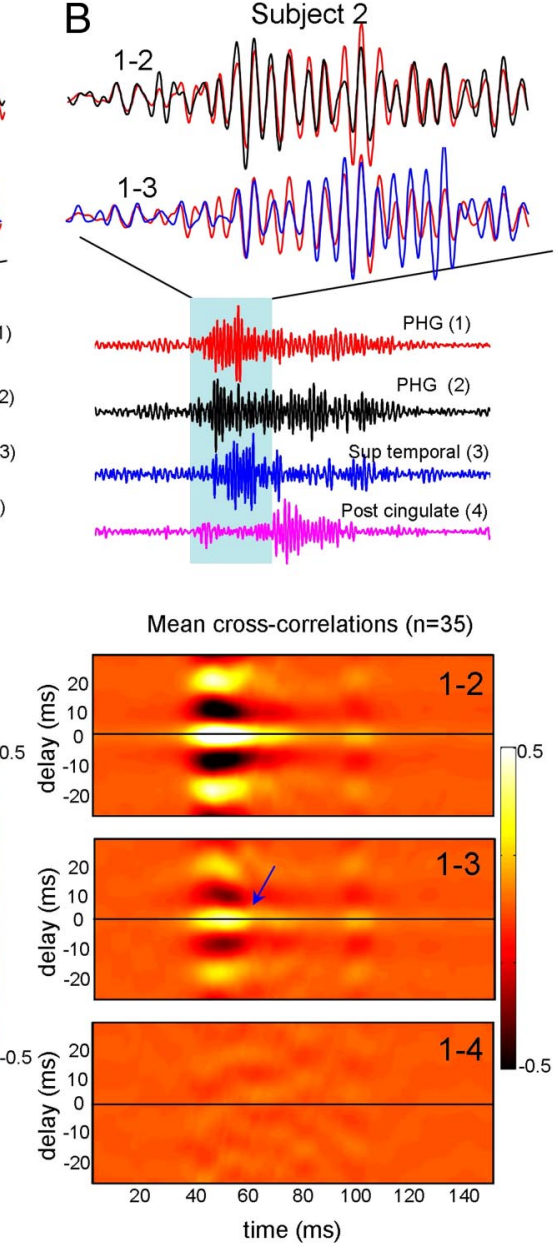

C

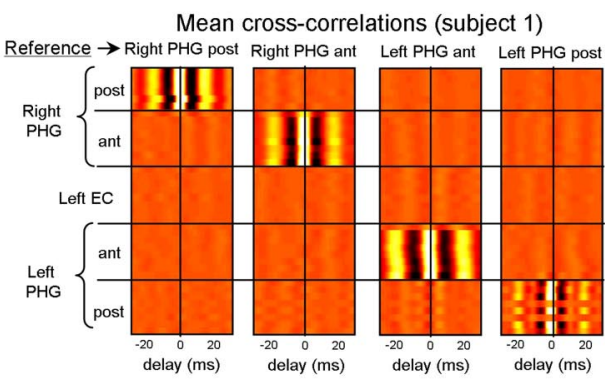

Mean cross-correlations (subject 2)

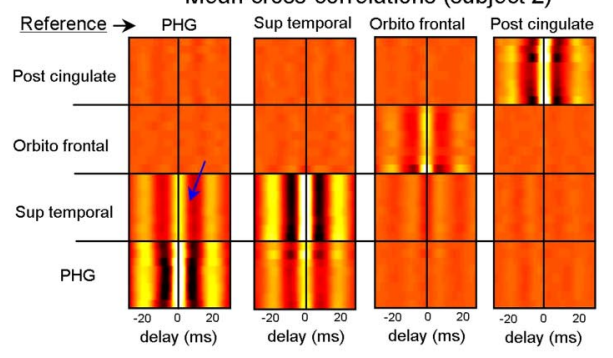

Mean cross-correlations (subject 3 )

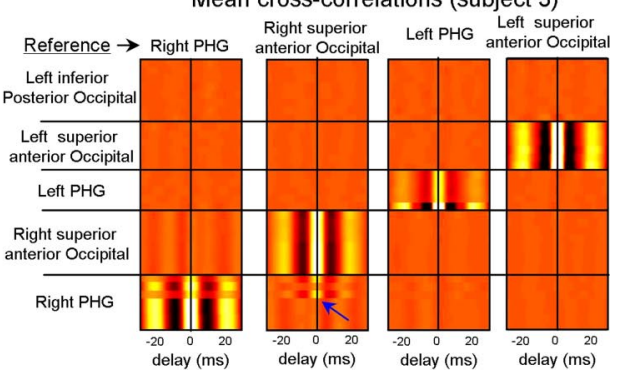

Figure 6. Spatial coherence of large-scale gamma oscillations. $\boldsymbol{A}, \boldsymbol{B}$, Single gamma pattern involving several spatially separated microelectrodes (1-4) (top) and their corresponding mean cross-correlograms (bottom). C, Mean cross-correlograms between one reference microelectrode and all other microelectrodes for three subjects during large-scale gamma patterns. Coherent gamma oscillations were occasionally observed between adjacent cortical areas over short distances $(<10 \mathrm{~mm})$ (patients 2 and 3 , blue arrows).

locking of a single unit within the range of high-gamma frequencies around $90 \mathrm{~Hz}$. Phase-related firing was also confirmed by spike-triggered averages of the LFP during gamma oscillations (Fig. 7Ci, top). For each neuron, spikes were phase locked most coherently in a narrow band of a particular gamma frequency, but over all recorded cells, multiple peaks of spike-LFP coherence could be observed across the whole gamma range (Fig. 7D). Furthermore, for the group of phase-locked cells, the distribution of their phases revealed that they fired preferentially during the troughs of the local field gamma oscillations (mean firing phase: $0.5 \pm 0.9 \mathrm{rad}$ ) (Fig. 7Cii,E).

\section{Synchronization of units during SWS gamma patterns}

Cross-correlograms were computed between all possible pairs of simultaneously recorded neurons during gamma oscillations in SWS. In order not to overestimate the number of random synchronous spikes due to the elevated firing rate, we used jitter techniques to infer millisecond-precise temporal synchrony (Hatsopoulos et al., 2003). Because the jittered datasets preserve firing rates on timescales much broader than that of the jitter interval (in this case, $5 \mathrm{~ms}$ ), the overall effect of the analysis is to identify those pairs that showed excessive cofiring at short latencies that cannot be accounted for by firing rates varying at timescales of tens of milliseconds. Figure $8 \mathrm{~A}$ illustrates all the neuronal pairs that showed significant coincident firing $(p=0.001)$ during gamma oscillations for a single subject. Despite the strong increase in all cellular discharges during gamma oscillations, only a very specific subset of cells (18 of 560 pairs in Fig. 8A) ( 2-3\% on average over four subjects) showed significant coincident firing during gamma oscillations. In four investigated subjects with a large number of single units $(>15)$ over several cortical structures $(>2)$, significant coincident firing could only be detected among neurons located within the same cortical area, corresponding to different microelectrodes emanating from the same electrode probe (separated by at least $500 \mu \mathrm{m}$, therefore recording non-overlapping cell populations). We performed the same analysis for baseline periods taken several seconds before the gamma oscillations and observed that a lower number of pairs exhibited significant coincident firing ( 10 of 560 pairs in Fig. 8 B). Over all investigated subjects, the number of pairs exhibiting synchronized activity was significantly increased, from $30 \%$ up to $200 \%$, during gamma oscillations compared with those of baseline periods (Fig. 8C). Overall, this suggests that patterns of neuronal synchronization between specific cells are significantly enhanced within a same cortical area during gamma oscillations. For pairs that showed significant coincident firing, we analyzed the corresponding phase relations of the LFP in the gamma range when cofiring occurred. Figure 8,D and $E$, indicates that cofiring mostly occurred when both cells fired around the troughs of the field gamma oscillations. This further suggests that the phase of 
A

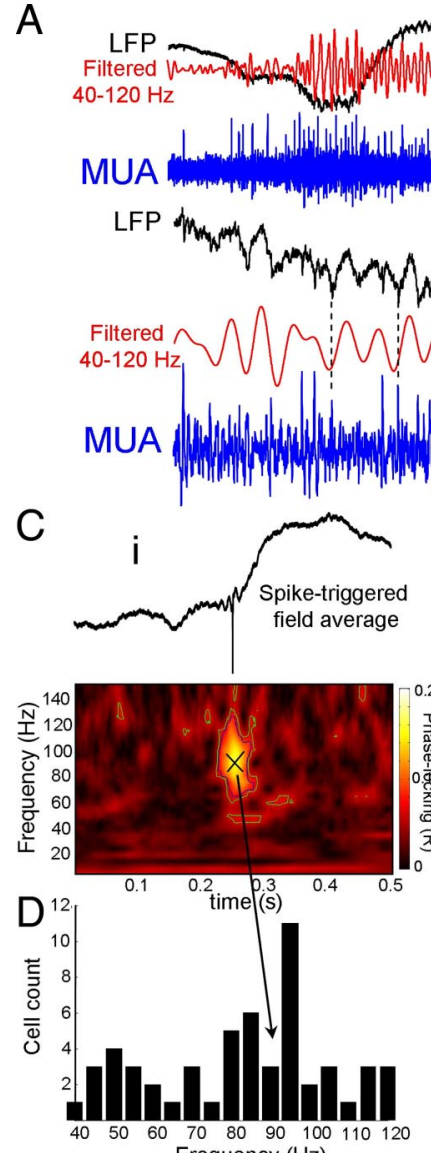

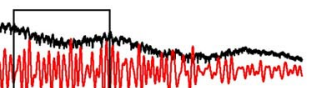

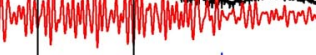

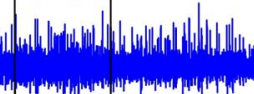
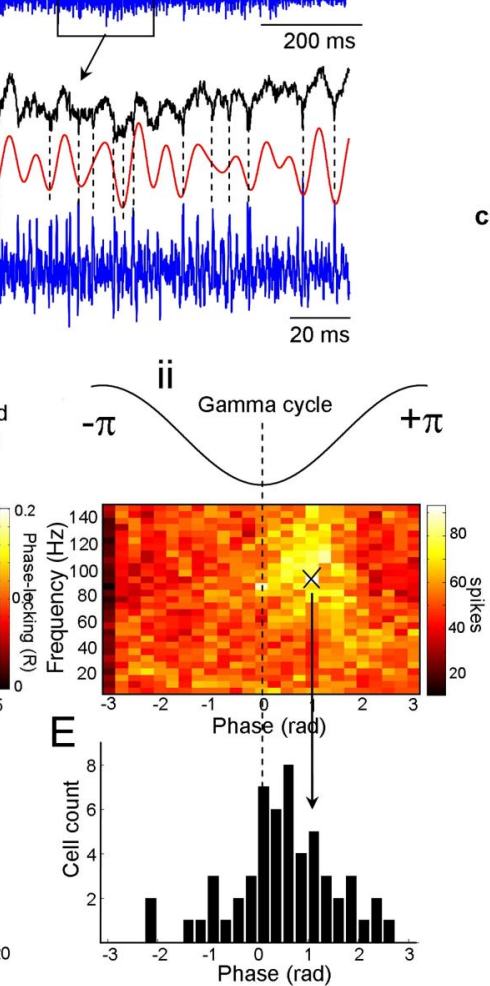
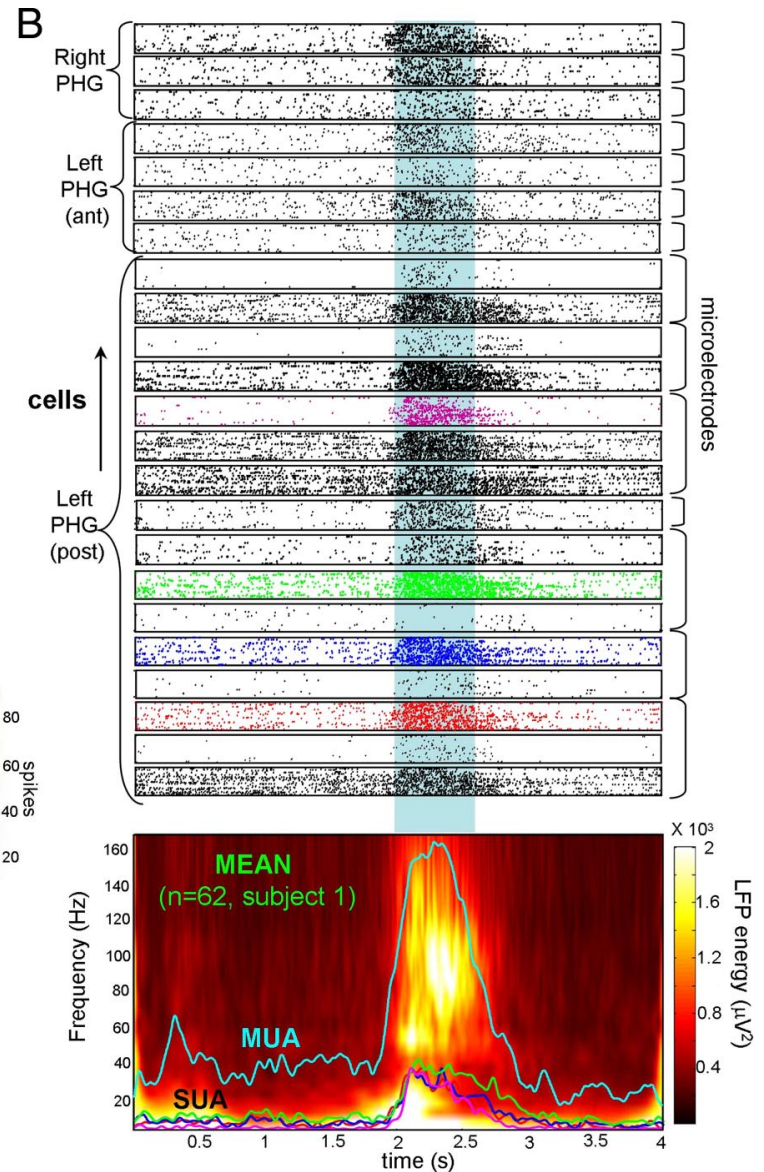

Figure 7. Gamma oscillation-related discharge of neurons. A, Strong MUAs were observed during gamma oscillation in LFPs. Note that spikes occurred preferentially around the troughs of the field gamma oscillations (subject 1, parahippocampal gyrus). B, Single-neuron firings of 23 cells in different cortical sites during gamma oscillations (subject 1). Note that the majority of single neurons exhibited a strong increase in spike discharges. The instantaneous frequencies of four cells (depicted with different colors) superimposed in the mean time-frequency map of the corresponding LFPs (bottom). Ci, Phase locking around $90 \mathrm{~Hz}$ between discharges from a single neuron and LFP oscillations. This phase locking was also confirmed by spike-triggered averages of the LFP during gamma oscillations (top). Cii, Phase distribution of the same neuron for each LFP frequency. $\boldsymbol{D}$, Group distribution of preferential LFP frequencies of phase-locked neurons in the gamma range (7 subjects). $\boldsymbol{E}$, Group distribution of preferential firing phases of phase-locked neurons in the gamma range. Note the tendency to fire preferentially during the troughs of the field gamma oscillations (7 subjects).

gamma oscillation may be a critical constraint for the dynamic coupling of interacting neurons.

\section{Discussion}

The present study explored the involvement of gamma oscillations in the human cortex using large-scale microelectrode recordings during the different stages of wakefulness and sleep. We reported that distinct narrow band oscillations in the low $(40-80$ $\mathrm{Hz}$ ) and high $(80-120 \mathrm{~Hz})$ gamma ranges were strongly expressed during SWS and often emerged at approximately the same time in multiple cortical areas over time windows of several hundreds of ms. These patterns were correlated with positive peaks of EEG slow oscillations and marked increases in local cellular discharges, suggesting that they were associated with cortical UP states.

\section{Multiple narrow-band gamma oscillations in the human cortex during sleep}

One important finding of our study is that gamma oscillations between 40 and $120 \mathrm{~Hz}$, generally regarded as characterizing states of brain arousal, also appear during natural sleep in the human cortex. The highest rates of oscillation detections were obtained in SWS, while the lowest rates were observed during waking. At first glance, our findings appear to contradict previous
EEG studies in both humans (Gross and Gotman, 1999; Cantero et al., 2004) and animal (Maloney et al., 1997) reporting that gamma activity is highest during wakefulness and REM, and lowest during SWS cycle. The most likely explanation for this discrepancy is the difference in methodology. First, we used microelectrodes that can record from local cortical generators rather than EEG recordings, which reflect the synchronization of large neuronal populations. Second, in previous studies, averaging of spectral activity over several minutes of recording cannot detect transient changes in oscillatory activities. Overall, our findings are consistent with other in vivo observations in animals (Steriade et al., 1996; Grenier et al., 2001; Isomura et al., 2006; Mukovski et al., 2007; Mena-Segovia et al., 2008), reporting neuronal responses in the beta/gamma bands $(>20 \mathrm{~Hz})$ during the depolarizing phase of slow sleep oscillations. Interestingly, in these animal studies, no discrete spectral peaks were reported in the high-frequency band, but rather a broad band continuum of activity in the low-gamma frequency range $(20-60 \mathrm{~Hz})$ (Steriade et al., 1996) and higher (80-200 Hz) (Grenier et al., 2001). Here, in contrast, we found that several discrete spectral peaks can be systematically identified in the low- and high-gamma ranges. These narrow-band oscillations could appear independently at overlapping locations but, most of the time, emerged together in 
A

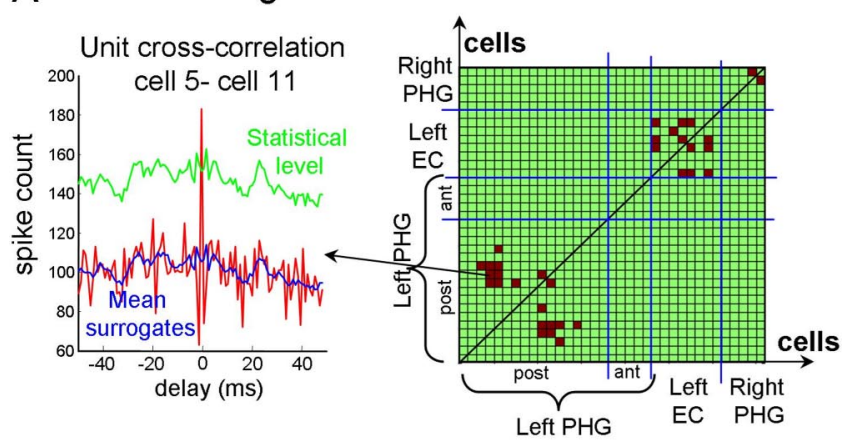

B

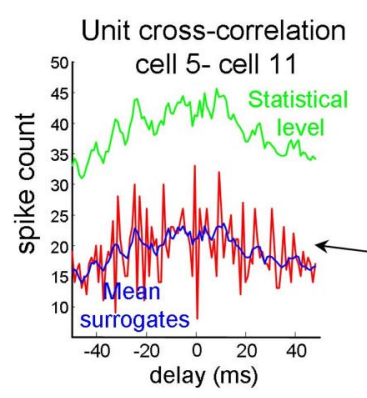

baseline

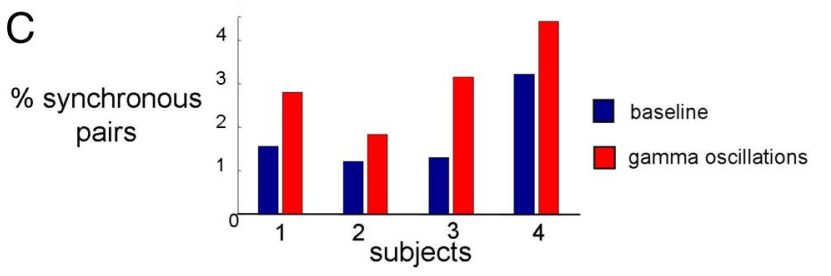

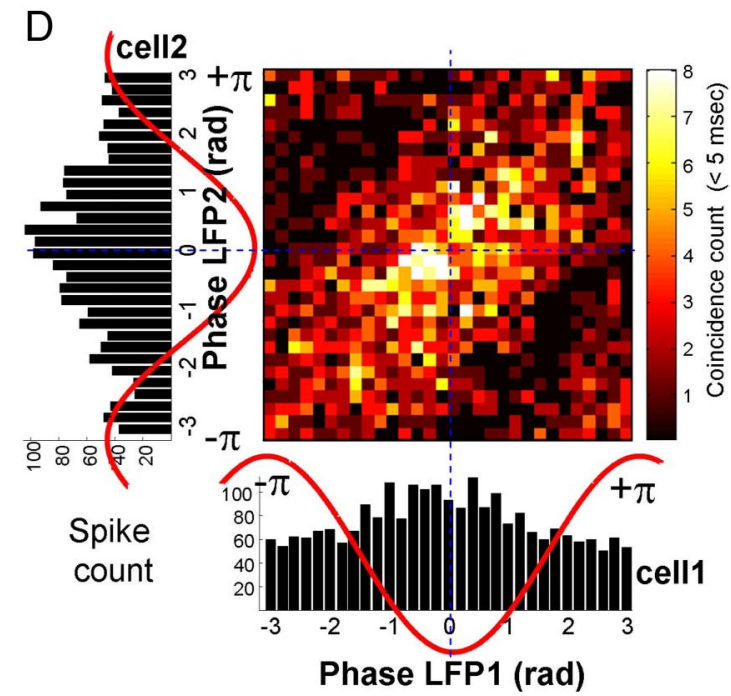

E

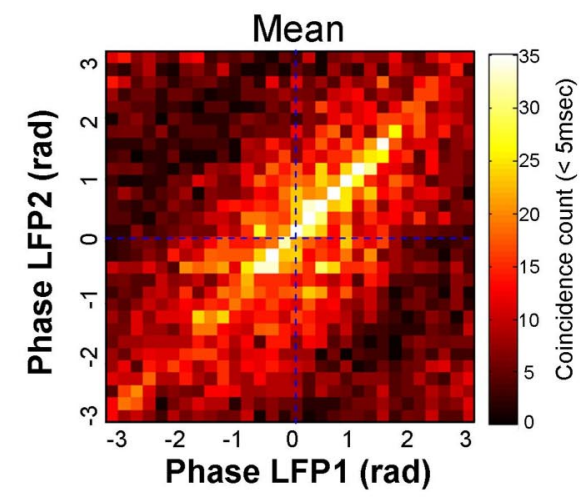

Figure 8. Synchronization of single units during gamma patterns. $A, B$, Right, Maps of the neuronal pairs that showed significant coincident firings during gamma oscillations and baseline (subject 1). Left, Cross-correlograms between a particular neuronal pair showing significant millisecond synchronization during gamma oscillations but not during baseline. $C$, Percentage of significant coincident firing computed between all possible pairs of simultaneously recorded neurons during baseline and gamma oscillations for four subjects. $D, E$, Phase values in the gamma range between two LFPs when cofiring occurred ( $<5 \mathrm{~ms}$ ) between two particular cells $(\boldsymbol{D})$ and corresponding average between 18 pairs of cells in four subjects $(\boldsymbol{E})$. Note that cofiring mostly occurred when both cells fired around the troughs of the field gamma oscillations.

mixed patterns. This suggests that independent gamma generators may exist within the human cortex. These findings may also relate to the proposal that, in the neocortex of human (Edwards et al., 2005) and monkey (Ray et al., 2008), low gamma (30-80 Hz) and high gamma $(80-140 \mathrm{~Hz})$ may reflect independent physiological mechanisms associated with different functions.

\section{Relations of gamma oscillations to epilepsy}

The present results were obtained in individuals with epilepsy, who may have abnormal synchrony during seizure-free periods in their global activity patterns. In particular, epileptiform discharges are often not limited to the primary epileptogenic region but can arise from larger areas that were recruited in previous seizures (de Curtis and Avanzini, 2001). Epileptic processes are also well known to be characterized by a general tendency of hypersynchronization and exaggeration of normal oscillations, as seen for example in the epileptic facilitation of sleep spindles (Steriade et al., 1994) or ripples (Clemens et al., 2007). However, epilepsy is unlikely to be the main source of the observed oscillations for the following reasons. First, gamma oscillations were associated with typical EEG slow waves fulfilling standard polysomnographic criteria. Second, in a majority of cases, cortical gamma oscillations were not coexistent with pathologic high-frequency oscillations $>250 \mathrm{~Hz}$ (Engel et al., 2009).
Third, for patients with mesial temporal lobe epilepsy, gamma oscillations were not temporally correlated with hippocampal interictal spike discharges.

\section{Large-scale spatial distribution of gamma patterns}

We reported that gamma oscillations can be recorded in all investigated cortical areas. Especially during SWS, gamma activities appeared at approximately the same time across many cortical regions and in homotopic sites forming large spatially coherent patterns. Because they were temporally correlated with positive peaks of EEG slow waves, this strongly suggests that this activity is partially synchronized with the cortical slow oscillation known to be synchronous over wide cortical territories and to involve multiple subsets of cortical structures (Massimini et al., 2004; Volgushev et al., 2006; Murphy et al., 2009). Consistent with this role, coherent slow fluctuations of gamma activity have been reported in the human sensory cortex that is significantly correlated across the two hemispheres (Nir et al., 2008).

\section{Local cortical coherence of gamma patterns}

In cats, coherence of high-frequency activity during slow oscillations was observed at distances between closely located cortical sites over a few millimeters with near zero phase lag but fell off 
then rapidly with distance greater than $5 \mathrm{~mm}$ (Steriade et al., 1996). In a similar way, we reported that gamma oscillations can be correlated with near zero phase lag $(<3 \mathrm{~ms})$ within a single cortical area $(<10 \mathrm{~mm})$ but long-range (between cortical regions) coherence fell off rapidly with distance. In particular, although gamma activities generally occurred almost simultaneously between the homotopic sides, there was no strict phase correlation across the hemispheres. As proposed before (Destexhe et al., 2007; Haider and McCormick, 2009), this short-range spatial confinement of fast rhythms during SWS likely exhibits characteristics similar to those observed during the waking state. Consistent with this hypothesis, neuroimaging studies (Dang-Vu et al., 2008) and high-resolution EEG imaging (Murphy et al., 2009) recently reported in humans that the response pattern related to SWS waves is close to the waking default mode network. These findings, together with our data, suggest that transient activations, especially in the gamma frequency range, during cortical UP states restore brief epochs of "microwake"-like activity patterns. These patterns could reflect recalled events experienced previously, which are "imprinted" in the network via synchronized network events that appear during sleep slow-wave complexes (Ji and Wilson, 2007; Luczak et al., 2007).

\section{Precise spike synchronization during gamma oscillations}

In the mammalian brain, synchrony of neuronal firing has been often observed to be associated with gamma oscillation during sensory information processing such as visual pattern recognition (Gray et al., 1989) or odor encoding (MacLeod and Laurent, 1996). Also, experimental findings demonstrated that the gamma frequency band of ongoing activity in the cortex could provide short "windows of opportunity" for interactions across remote subpopulations of cells (Chrobak and Buzsáki, 1998; Salinas and Sejnowski, 2001; Fries, 2005; Womelsdorf et al., 2007; Haider and McCormick, 2009). In particular, the negative phases of gamma activity provide temporal windows for putative interactions between interconnected neurons (Fries et al., 2007). Here, very similarly, our observations suggest that gamma oscillations during sleep may also briefly enhance local cortical communication by an increased millisecond precision of spike synchronization. These synchronizations may increase their output onto postsynaptic neurons, triggering precise firing sequence through local cortical networks (Luczak et al., 2007).

\section{The function of gamma coherence in sleep}

Slow-wave sleep, and particularly the cortical slow oscillation, is important for consolidating memory traces acquired during waking (Huber et al., 2004; Marshall et al., 2006). Gamma oscillations during sleep might also support these consolidation processes (Mölle et al., 2004), thus complementing the roles of gamma oscillations in encoding and retrieval of memory traces during wakefulness (Sejnowski and Paulsen, 2006; Montgomery and Buzsáki, 2007). Consistent with this, we reported that cofirings with millisecond precision between particular groups of cells are enhanced by gamma oscillations and provide means to initiate neuronal discharges on targets that would induce spike-timedependent plasticity in neocortical networks that may contribute to stabilize memories (Sejnowski and Paulsen, 2006). Furthermore, consistent with unit studies in animals (Isomura et al., 2006; Wolansky et al., 2006) or functional neuroimaging in humans (Dang-Vu et al., 2008), we also reported that gamma patterns have their strongest rates of detection and earliest activations in the parahippocampal gyrus. As a major relay between the hippocampus and the neocortex (Mohedano-Moriano et al., 2007), the parahippocampal cortex may play a critical role in sleep-associated consolidation of memory by transferring information between hippocampus and neocortex (Clemens et al., 2007, Axmacher et al., 2008). Indeed, we reported evidence of a probabilistic coupling between parahippocampal gamma oscillations and hippocampal ripple/sharp wave complexes, known to reactivate stored patterns of hippocampal activity (Wilson and McNaughton, 1994). In particular, in a significant number of cases, cortical gamma oscillations tended to occur within $100 \mathrm{~ms}$ after hippocampal ripple/sharp waves, suggesting an information flow from the hippocampus to the cortex. These observations are consistent with others reporting a temporal association between sharp waves and cortical slow waves in rodents (Battaglia et al., 2004) or humans (Clemens et al., 2007; Axmacher et al., 2008), suggesting a common driving influence of the slow oscillation on both hippocampal ripples and cortical gamma oscillations. We hypothesize that cortical gamma oscillations starting in correspondence with ripple/sharp wave complexes may be influenced by the reactivated information carried by the hippocampal sharp waves and could elicit coordinated retrieval of corresponding activity configurations stored in the cortex, especially in parahippocampal areas, that are indexed by the hippocampal cue.

\section{References}

Amzica F, Steriade M (1997) The K-complex: its slow (<1 Hz) rhythmicity and relation to delta waves. Neurology 49:952-959.

Axmacher N, Elger CE, Fell J (2008) Ripples in the medial temporal lobe are relevant for human memory consolidation. Brain 131:1806-1817.

Bartos M, Vida I, Jonas P (2007) Synaptic mechanisms of synchronized gamma oscillations in inhibitory interneuron networks. Nat Rev Neurosci 8:45-56.

Battaglia FP, Sutherland GR, McNaughton BL (2004) Hippocampal sharp wave bursts coincide with neocortical "up-state" transitions. Learn Mem 11:697-704.

Bragin A, Engel J Jr, Wilson CL, Fried I, Buzsáki G (1999) High-frequency oscillations in human brain. Hippocampus 9:137-142.

Bragin A, Mody I, Wilson CL, Engel J Jr (2002) Local generation of fast ripples in epileptic brain. J Neurosci 22:2012-2021.

Buzsáki G (2004) Large-scale recording of neuronal ensembles. Nat Neurosci 7:446-451.

Cantero JL, Atienza M, Madsen JR, Stickgold R (2004) Gamma EEG dynamics in neocortex and hippocampus during human wakefulness and sleep. Neuroimage 22:1271-1280.

Cash SS, Halgren E, Dehghani N, Rossetti AO, Thesen T, Wang C, Devinsky O, Kuzniecky R, Doyle W, Madsen JR, Bromfield E, Eross L, Halász P, Karmos G, Csercsa R, Wittner L, Ulbert I (2009) The human K-complex represents an isolated cortical down-state. Science 324:1084-1087.

Chrobak JJ, Buzsáki G (1998) Operational dynamics in the hippocampalentorhinal axis. Neurosci Biobehav Rev 22:303-310.

Clemens Z, Mölle M, Eross L, Barsi P, Halász P, Born J (2007) Temporal coupling of parahippocampal ripples, sleep spindles and slow oscillations in humans. Brain 130:2868-2878.

Compte A, Reig R, Descalzo VF, Harvey MA, Puccini GD, Sanchez-Vives MV (2008) Spontaneous high-frequency $(10-80 \mathrm{~Hz})$ oscillations during UP states in the cerebral cortex in vitro. J Neurosci 28:13828-13844.

Dang-Vu TT, Schabus M, Desseilles M, Albouy G, Boly M, Darsaud A, Gais S, Rauchs G, Sterpenich V, Vandewalle G, Carrier J, Moonen G, Balteau E, Degueldre C, Luxen A, Phillips C, Maquet P (2008) Spontaneous neural activity during human slow wave sleep. Proc Natl Acad Sci USA 105:15160-15165.

de Curtis M, Avanzini G (2001) Interictal spikes in focal epileptogenesis. Prog Neurobiol 63:541-567.

Destexhe A, Hughes SW, Rudolph M, Crunelli V (2007) Are corticothalamic 'UP' states fragments of wakefulness? Trends Neurosci 30:334-342.

Dickson CT, Biella G, de Curtis M (2003) Slow periodic events and their transition to gamma oscillations in the entorhinal cortex of the isolated guinea pig brain. J Neurophysiol 90:39-46.

Edwards E, Soltani M, Deouell LY, Berger MS, Knight RT (2005) High 
gamma activity in response to deviant auditory stimuli recorded directly from human cortex. J Neurophysiol 94:4269-4280.

Engel J Jr, Bragin A, Staba R, Mody I (2009) High-frequency oscillations: what is normal and what is not? Epilepsia 50:598-604.

Fisher N (1993) Statistical analysis of circular data. Cambridge, UK: Cambridge UP.

Fried I, MacDonald KA, Wilson CL (1997) Single neuron activity in human hippocampus and amygdala during recognition of faces and objects. Neuron 18:753-765.

Fries P (2005) A mechanism for cognitive dynamics: neuronal communication through neuronal coherence. Trends Cogn Sci 9:474-480.

Fries P, Reynolds JH, Rorie AE, Desimone R (2001) Modulation of oscillatory neuronal synchronization by selective visual attention. Science 291:1560-1563.

Fries P, Nikolić D, Singer W (2007) The gamma cycle. Trends Neurosci 30:309-316.

Gray CM, König P, Engel AK, Singer W (1989) Oscillatory responses in cat visual cortex exhibit inter-columnar synchronization which reflects global stimulus properties. Nature 338:334-337.

Grenier F, Timofeev I, Steriade M (2001) Focal synchronization of ripples $(80-200 \mathrm{~Hz})$ in neocortex and their neuronal correlates. J Neurophysiol $86: 1884-1898$.

Gross DW, Gotman J (1999) Correlation of high frequency oscillations with the sleep-wake cycle and cognitive activity in humans. Neuroscience 94:1005-1018.

Haider B, McCormick DA (2009) Rapid neocortical dynamics: cellular and network mechanisms. Neuron 62:171-189.

Harris KD, Hirase H, Leinekugel X, Henze DA, Buzsáki G (2001) Temporal interaction between single spikes and complex spike bursts in hippocampal pyramidal cells. Neuron 32:141-149.

Hasenstaub A, Shu Y, Haider B, Kraushaar U, Duque A, McCormick DA (2005) Inhibitory postsynaptic potentials carry synchronized frequency information in active cortical networks. Neuron 47:423-435.

Hatsopoulos N, Geman S, Amarasingham A, Bienenstock E (2003) At what time scale does the nervous system operate? Neurocomputing 52-54:25-29.

Huber R, Ghilardi MF, Massimini M, Tononi G (2004) Local sleep and learning. Nature 430:78-81.

Isomura Y, Sirota A, Ozen S, Montgomery S, Mizuseki K, Henze DA, Buzsáki G (2006) Integration and segregation of activity in entorhinal-hippocampal subregions by neocortical slow oscillations. Neuron 52:871-882.

Jacobs J, Kahana MJ, Ekstrom AD, Fried I (2007) Brain oscillations control timing of single-neuron activity in humans. J Neurosci 27:3839-3844.

Ji D, Wilson MA (2007) Coordinated memory replay in the visual cortex and hippocampus. Nat Neurosci 10:100-107.

Le Van Quyen M, Bragin A (2007) Analysis of dynamic brain oscillations: methodological advances. Trends Neurosci 30:365-373.

Le Van Quyen M, Bragin A, Staba R, Crépon B, Wilson CL, Engel J Jr (2008) Cell type-specific firing during ripple oscillations in the hippocampal formation of humans. J Neurosci 28:6104-6110.

Luczak A, Barthó P, Marguet SL, Buzsáki G, Harris KD (2007) Sequential structure of neocortical spontaneous activity in vivo. Proc Natl Acad Sci U S A 104:347-352.

MacLeod K, Laurent G (1996) Distinct mechanisms for synchronization and temporal patterning of odor-encoding cell assemblies. Science 274:976-979.

Maloney KJ, Cape EG, Gotman J, Jones BE (1997) High frequency gamma electroencephalogram activity in association with sleep-wake states and spontaneous behaviors in the rats. Neuroscience 76:541-555.

Marshall L, Helgadóttir H, Mölle M, Born J (2006) Boosting slow oscillations during sleep potentiates memory. Nature 444:610-613.

Massimini M, Huber R, Ferrarelli F, Hill S, Tononi G (2004) The sleep slow oscillation as a traveling wave. J Neurosci 24:6862-6870.

Mena-Segovia J, Sims HM, Magill PJ, Bolam JP (2008) Cholinergic brainstem neurons modulate cortical gamma activity during slow oscillations. J Physiol 586:2947-2960.

Mohedano-Moriano A, Pro-Sistiaga P, Arroyo-Jimenez MM, Artacho-Pérula E, Insausti AM, Marcos P, Cebada-Sánchez S, Martínez-Ruiz J, Muñoz M,
Blaizot X, Martinez-Marcos A, Amaral DG, Insausti R (2007) Topographical and laminar distribution of cortical input to the monkey entorhinal cortex. J Anat 211:250-260.

Mölle M, Marshall L, Gais S, Born J (2004) Learning increases human electroencephalographic coherence during subsequent slow sleep oscillations. Proc Natl Acad Sci U S A 101:13963-13968.

Montgomery SM, Buzsáki G (2007) Gamma oscillations dynamically couple hippocampal CA3 and CA1 regions during memory task performance. Proc Natl Acad Sci U S A 104:14495-14500.

Mukovski M, Chauvette S, Timofeev I, Volgushev M (2007) Detection of active and silent states in neocortical neurons from the field potential signal during slow-wave sleep. Cereb Cortex 17:400-414.

Murphy M, Riedner BA, Huber R, Massimini M, Ferrarelli F, Tononi G (2009) Source modeling sleep slow waves. Proc Natl Acad Sci U S A 106:1608-1613.

Nicolelis MA, Dimitrov D, Carmena JM, Crist R, Lehew G, Kralik JD, Wise SP (2003) Chronic, multisite, multielectrode recordings in macaque monkeys. Proc Natl Acad Sci U S A 100:11041-11046.

Nir Y, Mukamel R, Dinstein I, Privman E, Harel M, Fisch L, Gelbard-Sagiv H, Kipervasser S, Andelman F, Neufeld MY, Kramer U, Arieli A, Fried I, Malach R (2008) Interhemispheric correlations of slow spontaneous neuronal fluctuations revealed in human sensory cortex. Nat Neurosci 11:1100-1108.

Nowak LG, Sanchez-Vives MV, McCormick DA (1997) Influence of low and high frequency inputs on spike timing in visual cortical neurons. Cereb Cortex 7:487-501.

Ray S, Crone NE, Niebur E, Franaszczuk PJ, Hsiao SS (2008) Neural correlates of high-gamma oscillations $(60-200 \mathrm{~Hz})$ in macaque local field potentials and their potential implications in electrocorticography. J Neurosci 28:11526-11536.

Rechtschaffen A, Kales A (1968) A manual of standardized terminology, techniques and scoring system for sleep stages of human subjects. Washington, DC: National Institutes of Health.

Salinas E, Sejnowski TJ (2001) Correlated neuronal activity and the flow of neural information. Nat Rev Neurosci 2:539-550.

Sejnowski TJ, Destexhe A (2000) Why do we sleep? Brain Res 886:208-223.

Sejnowski TJ, Paulsen O (2006) Network oscillations: emerging computational principles. J Neurosci 26:1673-1676.

Singer W, Gray CM (1995) Visual feature integration and the temporal correlation hypothesis. Annu Rev Neurosci 18:555-586.

Staba RJ, Wilson CL, Bragin A, Fried I, Engel J Jr (2002) Sleep states differentiate single neuron activity recorded from human epileptic hippocampus, entorhinal cortex, and subiculum. J Neurosci 22:5694-5704.

Staba RJ, Wilson CL, Bragin A, Jhung D, Fried I, Engel J Jr (2004) Highfrequency oscillations recorded in human medial temporal lobe during sleep. Ann Neurol 56:108-115.

Steriade M (2000) Corticothalamic resonance, states of vigilance and mentation. Neuroscience 101:243-276.

Steriade M, Nuñez A, Amzica F (1993) A novel slow ( $<1 \mathrm{~Hz}$ ) oscillation of neocortical neurons in vivo: depolarizing and hyperpolarizing components. J Neurosci 13:3252-3265.

Steriade M, Contreras D, Amzica F (1994) Synchronized sleep oscillations and their paroxysmal developments. Trends Neurosci 17:199-208.

Steriade M, Amzica F, Contreras D (1996) Synchronization of fast (30-40 $\mathrm{Hz}$ ) spontaneous cortical rhythms during brain activation. J Neurosci 16:392-417.

Volgushev M, Chauvette S, Mukovski M, Timofeev I (2006) Precise longrange synchronization of activity and silence in neocortical neurons during slow-wave sleep. J Neurosci 26:5665-5672.

Wilson MA, McNaughton BL (1994) Reactivation of hippocampal ensemble memories during sleep. Science 265:676-679.

Wolansky T, Clement EA, Peters SR, Palczak MA, Dickson CT (2006) The hippocampal slow oscillation: a novel EEG state and its coordination with ongoing neocortical activity. J Neurosci 26:6213-6229.

Womelsdorf T, Schoffelen JM, Oostenveld R, Singer W, Desimone R, Engel AK, Fries P (2007) Modulation of neuronal interactions through neuronal synchronization. Science 316:1609-1612. 